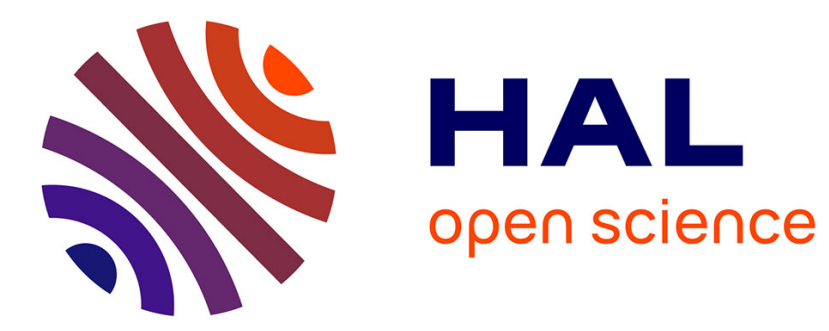

\title{
OsJAZ9 overexpression modulates jasmonic acid biosynthesis and potassium deficiency responses in rice
} Ajit Pal Singh, Bipin K. Pandey, Poonam Mehra, Thierry Heitz, Jotender Giri

\section{To cite this version:}

Ajit Pal Singh, Bipin K. Pandey, Poonam Mehra, Thierry Heitz, Jotender Giri. OsJAZ9 overexpression modulates jasmonic acid biosynthesis and potassium deficiency responses in rice. Plant Molecular Biology, 2020, 104, pp.397-410. 10.1007/s11103-020-01047-2 . hal-03039565

\section{HAL Id: hal-03039565 https://hal.science/hal-03039565}

Submitted on 3 Dec 2020

HAL is a multi-disciplinary open access archive for the deposit and dissemination of scientific research documents, whether they are published or not. The documents may come from teaching and research institutions in France or abroad, or from public or private research centers.
L'archive ouverte pluridisciplinaire HAL, est destinée au dépôt et à la diffusion de documents scientifiques de niveau recherche, publiés ou non, émanant des établissements d'enseignement et de recherche français ou étrangers, des laboratoires publics ou privés. 
1 OsJAZ9 overexpression modulates jasmonic acid biosynthesis and potassium deficiency

2 responses in rice

3 Ajit Pal Singh $\dagger^{1}$, Bipin K. Pandey $\dagger^{1,2}$, Poonam Mehra ${ }^{1}$, Thierry Heitz ${ }^{3}$ \& Jitender Giri ${ }^{1 *}$

4

$5 \quad{ }^{1}$ National Institute of Plant Genome Research, Aruna Asaf Ali Marg, New Delhi-110067, India

$6 \quad{ }^{2}$ Plant and Crop Science Division, School of Biosciences, University of Nottingham, UK

$7 \quad{ }^{3}$ Institut de Biologie Moléculaire des Plantes (IBMP) du CNRS, Université de Strasbourg, Strasbourg,

8 France

$9+$ Equal contribution

10 *Author for correspondence

11 Jitender Giri

12 jitender@nipgr.ac.in

13

91-11-26735227

14 


\section{Abstract:}

Potassium (K) represents up to $10 \%$ of the plant's total dry biomass, and its deficiency makes plants highly susceptible to both abiotic and biotic stresses. $\mathrm{K}$ shortage results in the inhibition of root and shoots growth, but the underlying mechanism of this response is unclear. Our RNA-Seq and qPCR analysis suggested leading roles for JA pathway genes under $\mathrm{K}$ deficiency in rice. Notably, $\mathrm{K}$ deficiency and JA application produced similar phenotypic and transcriptional responses. Here, we integrated molecular, physiological and morphological studies to analyze the role of OSJAZ9 in JA homeostasis and $\mathrm{K}$ deficiency responses. We raised OSJAZ9 over-expression, knockdown, transcriptional reporter, translational reporter and $\mathrm{C}$-terminal deleted translational reporter lines in rice to establish the role of JA signaling in $\mathrm{K}$ ion homeostasis. JA profiling revealed significantly increased JA-Ile levels in OsJAZ9 OE lines under K deficiency. Furthermore, we established that OsJAZ9 overexpression and knockdown result in $\mathrm{K}$ deficiency tolerance and sensitivity, respectively, by modulating various $\mathrm{K}$ transporters and root system architecture. Our data provide evidence on the crucial roles of OsJAZ9 for improving $\mathrm{K}$ deficiency tolerance in rice by altering JA levels and JA responses.

Keywords: Abiotic stress, Potassium deficiency, JAZ repressor, Root system architecture, Lateral roots 
Rice is a mainstay for global food security. The two-third population of the world is dependent on rice as a staple food crop. Soil nutrient deficiency is one of the primary limitations of rice production. Notably, Potassium $(\mathrm{K})$ is one of the most important and abundant macronutrients in plants, which can comprise up to $10 \%$ of the plant's total dry weight (Leigh \& Wyn Jones, 1984). Its physiological functions fall into two categories; the first requires a high and relatively stable concentration of $\mathrm{K}^{+}$to regulate the osmotic potential of the cell and activation of many enzymes involved in respiration and photosynthesis ( $\mathrm{K}^{+}$act as a cofactor for many enzymes). The second category is based on the high mobility of $\mathrm{K}^{+}$ions resulting in osmotic changes, which regulate the stomatal movement (Sawhney \& Zelitch, 1969; Terry \& Ulrich, 1973) and phloem transport (Mengel and Viro, 1974). $\mathrm{K}^{+}$is also involved in key cellular processes such as energy production, cell expansion and balancing the counter flux of charges across the membranes (Tester and Blatt, 1989; Wu et al., 1991; Elumalai et al., 2002). Hence, its deficiency affects fitness and overall growth as K deficient plants are more susceptible to salt (Kaya et al., 2006), drought (Egilla et al., 2001; Wang et al., 2013), chilling (Kant and Kafkafi, 2002) and biotic stresses (Hardter, 2002; Sarwar, 2012).

Although $\mathrm{K}$ is among the most abundant minerals in the soil; its availability to plants is limited because most of the soil $\mathrm{K}$ (about 98\%) is in bound forms where its release into the soil solution is far slower than the rate of acquisition by roots (Sparks, 1987). Notably, a very little fraction of soil $\mathrm{K}$ is present in the soil solution or exchangeable form whose availability depends on multiple factors like soil pH (Rich and Black, 1964; Rich, 1964), presence of other monovalent cations like $\mathrm{Na}^{+}$and $\mathrm{NH}_{4}^{+}$(Qi and Spalding, 2004) and the type of soil particles (Sparks, 1987). K deficiency results in both root (Singh et al., 2015) and shoot (Shankar et al., 2013) growth inhibition. In roots, it strongly impairs primary root growth (Gruber et al., 2013); a similar phenotype is also observed on exogenous Jasmonic Acid (JA) application (Staswick et al., 1992; Cai et al., 2014). Further, a few transcriptome studies have shown many JA signaling genes upregulated under K deficiency in rice (Ma et a., 2012; Shankar et al., 2013; Takehisa et al., 2013). Interestingly, a substantial part of K-responsive transcriptome either was absent or replaced in the Arabidopsis JA receptor, coil-16 mutant (Armengaud et al., 2004) which indicates active roles of JA signaling in $\mathrm{K}$ deficiency responses. Moreover, a significant number of genes were also found commonly upregulated by exogenous JA treatment and K deficiency in rice (Kobayashi et al., 2016), which further indicates crosstalk between JA signaling and $\mathrm{K}$ deficiency response. 
JA and its derivatives commonly called Jasmonates (JAs) are a group of oxylipin-derived phytohormones, which are synthesized in response to a large number of biotic and abiotic stresses in plants (Feys et al., 1994; Heitz et al., 2016; Kazan, 2015; Riemann et al., 2015). Understanding of JA functions is most advanced in Arabidopsis, but the overall architecture has been found conserved in all examined land plants. JA signaling has been the focus of intensive research in rice and regulates many traits of agronomic importance (Reimann et al., 2015; Nguyen et al., 2019). In absence of JA, JA-regulated genes are maintained silent by socalled JASMONATE ZIM (JAZ) protein repressors that bind to and block the action of bHLH transcription factors MYC2/3/4, with the involvement of additional repressors. Upon stress of developmental cues, the bioactive conjugate jasmonoyl-isoleucine JA-Ile is formed and triggers the assembly of the co-receptor JAZ-COI1 (Sheard et al., 2010). COI1 (CORONATINE INSENSITIVE 1), is the F-box component of the co-receptor, that will be recruited into the ubiquitin E3 ligase complex $\mathrm{SCF}^{\mathrm{CO}}{ }^{1}$ that targets JAZ protein for $26 \mathrm{~S}$ proteasome-mediated degradation (Wasternack and Hause, 2013). JAZ destabilisation derepresses a myriad of transcription factors allows the transcription of numerous JA responsive genes depending on organ or stimulus (Wasternack and Hause, 2013). JAZs form a small family of 15 members in rice (Ye et al., 2009), and play roles in floral development, salt, and biotic stress tolerance (Fu et al., 2017). However, their functions in nutritional, particularly $\mathrm{K}$ deficiency response, remain elusive. Here, we examined the functions of a particular JAZ isoform, OsJAZ9, because OsJAZ9 was the most downregulated $J A Z$ gene during $\mathrm{K}$ deficiency among all the isoforms (Singh et al., 2015). Further, OsJAZ9 also influenced the rice response to $\mathrm{NaCl}$ stress which also involves $\mathrm{K}$ homeostasis (Wu et al., 2015). We show that JA-Ile levels are enhanced under $\mathrm{K}$ deficiency in rice and this response is further elevated in rice on OsJAZ9 overexpression. Also, we demonstrate that overexpression of OSJAZ9 enhanced JA signaling, and this was correlated with improved $\mathrm{K}$ deficiency tolerance.

\section{Materials and Methods}

\section{Plant growth conditions}

Rice seeds var. PB1 (Indica) were surface-sterilized with $0.1 \% \mathrm{HgCl}_{2}$ for $10 \mathrm{~min}$ and then washed with sterile water five times. After that, seeds were germinated on autoclaved wet tissue paper for two days in the dark, followed by two days in the light. Equally germinated seeds were then transferred to full strength liquid Yoshida media $\left(\mathrm{pH}\right.$ 5.0-5.5) with $\mathrm{NH}_{4} \mathrm{NO}_{3}(1.40$ $\mathrm{mM}), \mathrm{NaH}_{2} \mathrm{PO}_{4}(0.32 \mathrm{mM}), \mathrm{K}_{2} \mathrm{SO}_{4}(0.51 \mathrm{mM}), \mathrm{CaCl}_{2} .2 \mathrm{H}_{2} \mathrm{O}(1 \mathrm{mM}), \mathrm{MgSO}_{4} .7 \mathrm{H}_{2} \mathrm{O}(1.7 \mathrm{mM})$, 

$\mu \mathrm{M})$, Citric Acid $(70.75 \mu \mathrm{M})$, Na-Fe-EDTA $(60 \mu \mathrm{M})$ and $\mathrm{MnCl}_{2} .4 \mathrm{H}_{2} \mathrm{O}(9.46 \mu \mathrm{M})$. The concentration of $\mathrm{K}_{2} \mathrm{SO}_{4}$ was adjusted as required to make-up the desired concentration of $\mathrm{K}$. All the experiments were carried out in a controlled growth chamber maintained at $16 \mathrm{~h}$ photoperiod, $30^{\circ} \mathrm{C}$ day and $28^{\circ} \mathrm{C}$ night temperature, $280-300 \mu \mathrm{M}$ photons $/ \mathrm{m} 2 / \mathrm{sec}$ photon density and $70 \%$ relative humidity.

\section{qRT-PCR analysis}

117

118

119

120

Total RNA was extracted from seedlings of the desired age using Trizol reagent and cDNA synthesis was performed as described (Mehra et al., 2017). Gene-specific primers were designed from CDS (retrieved from Rice Gene Annotation Project) using PRIMER EXPRESS version 3.0 (PE Applied Biosystems TM, USA) with default parameters. SYBR ${ }^{\circledR}$ Green Master Mix was used to quantify the DNA product in Applied Biosystems 7500 Fast RealTime PCR. For the Relative gene expression analysis, we used the $\Delta \Delta \mathrm{Ct}$ method taking Ubiquitin5 (Os01g0328400) or Actin1 (Os03g0718100) as endogenous control (Mehra et al., 2016).

\section{Measurement of $\mathrm{K}$ and $\mathrm{Na}$}

Root and shoot tissues were harvested separately and repeatedly washed with milliQ water. Dried $50 \mathrm{mg}$ tissue was ground and digested in di-acid mixture $(20 \mathrm{ml})$ containing HNO3 and $\mathrm{HClO} 4$ acid (9:4 ratio) using a hot plate digestion system. The diluted cooled digest was filtered and stored in polypropylene bottles with a final volume of $50 \mathrm{ml}$. Analysis of $\mathrm{Na}$ and $\mathrm{K}$ content was performed using a flame photometer (Systronics Flame Photometer 128 $\mu \mathrm{C}$ ).

\section{Generation of transgenic lines}

Full-length cDNA (AK070649) of OsJAZ9 was amplified using gene-specific primers (Supplemental Table S1) and cloned under ZmUbil promoter in gateway-compatible overexpression vector as described (Pandey et al., 2017). For raising RNAi transgenics of OSJAZ9, $416 \mathrm{bp}$ region of OSJAZ9 cDNA was amplified from the cDNA region encompassing 3'UTR and cloned in $p A N I C 8 b$ vector (Mann et al., 2012) by Gateway Technology. To generate p35S:OsJAZ9_GUS and p35S:OSJAZ9DC_GUS lines, OsJAZ9 coding region and OsJAZ9AC (deleted 65 amino acids including jas motif from the C-terminal of OsJAZ9) cDNAs were amplified using gene-specific primers, and cloned into pCAMBIA1301. 
Overexpression, RNAi, p35S:OsJAZ9_GUS and p35S:OsJAZ9DC_GUS transgenic rice lines were raised as described (Mehra et al., 2017).

142 For generating OsJAZ9 promoter lines ( $p O s J A Z 9: G U S), 1495 \mathrm{~kb}$ upstream region of the gene CDS (OsJAZ9) was cloned in pMDC163 driving GUS reporter gene. Regenerated plants were selected on hygromycin $(50 \mu \mathrm{g} / \mathrm{ml})$ and screened by qRT-PCR and GUS histochemical staining. All the experiments were performed in T3 homozygous transgenic lines.

\section{Analysis of GUS Activity}

Newly emerged roots were selected for histochemical staining, i.e. GUS activity. Samples were harvested at indicated time points and immersed in GUS buffer (1 mM 5-bromo-4-chloro-3indolyl- $\beta$-glucuronide sodium salt in $50 \mathrm{mM}$ sodium phosphate, $\mathrm{pH} 7.0$, ten $\mathrm{mM}$ Na-EDTA, $0.5 \mathrm{mM}$ ferricyanide, $0.5 \mathrm{mM}$ ferrocyanide, and $0.1 \%$ Triton X-100), and were incubated at $37^{\circ} \mathrm{C}$ for overnight. Chlorophyll was cleared from plant tissues by immersing them in $70 \%$ ethanol, overnight. For detection of OsJAZ9 degradation in-vivo, roots from transgenic lines containing p35S:OsJAZ9_GUS and p35S:OSJAZ9DC_GUS plants were treated with MeJA $(100 \mu \mathrm{M})$ with or without the proteasome inhibitor MG132 $(100 \mu \mathrm{M})$ for $1 \mathrm{~h}$. Histochemical GUS staining of roots was performed to visualize the abundance/degradation of OsJAZ9 and OsJAZ9 $\triangle$ C. GUS signal was observed under a stereo-zoom microscope. Fifteen-days-old seedlings of p35S:OsJAZ9_GUS and p35S:OsJAZ9AC_GUS transgenic lines were subjected to $\mathrm{K}$ deficiency $\left(4.08 \mu \mathrm{M}\right.$ external $\left.\mathrm{K}^{+}\right)$and root samples were harvested on indicated time points and immersed in GUS buffer. $p O S J A Z 9: G U S$ transgenic lines were subjected to K deficiency $\left(0 \mu \mathrm{M}\right.$ external $\left.\mathrm{K}^{+}\right)$and root samples were harvested on indicated time points and immersed in GUS buffer.

\section{Subcellular localization of OsJAZ9}

In-silico prediction of OsJAZ9 was analyzed with the help of Plant-PLoc database (http://www.csbio.sjtu.edu.cn/cgi-bin/PlantmPLoc.cgi). For in-plants validation, CDS of OsJAZ9 was cloned into pSITE3CA vector to produce YFP:OsJAZ9 fusion protein. Subcellular localization was analyzed in onion epidermal cells using the particle bombardment method (Singh et al., 2015). YFP fluorescence was visualized under a fluorescence microscope 168 (Nikon Eclipse 80i). 
Uniformly, germinated seedlings were transferred to Yoshida liquid media and allowed to grow for 10 days under normal conditions. After 10 days of growth, $10 \mu \mathrm{M}$ MeJA dissolved in DMSO was added to one set of plants (including WT, RN12, and OE10) while another set was supplied with DMSO alone (mock). pH was maintained at 5.4 and the media was replenished after five days. Root growth inhibition was analyzed after 10 days of MeJA treatment by calculating the percentage change in root growth between DMSO and MeJA treated plants.

\section{Exogenous MeJA treatment to analyze the impact on $\mathrm{K}$ deficiency}

To investigate the impact of exogenous application of MeJA on $\mathrm{K}$ deficiency, uniformly germinated WT rice seedlings were transferred to hydroponic (Yoshida) media with $4.08 \mu \mathrm{M}$ $\mathrm{K}_{2} \mathrm{SO}_{4}, 8.16 \mu \mathrm{M} \mathrm{K}_{2} \mathrm{SO}_{4}$ and $510 \mu \mathrm{M} \mathrm{K}_{2} \mathrm{SO}_{4}$ for 5 days and then equal number of seedlings were transferred to $0 \mu \mathrm{M}, 0.1 \mu \mathrm{M}, 0.01 \mu \mathrm{M}$ and $0.001 \mu \mathrm{M}$ MeJA with pre-existing $\mathrm{K}_{2} \mathrm{SO}_{4}$ concentrations for 10 days. These seedlings were analyzed for root length, shoot length and lateral root length. After this analysis, the same seedlings were transferred to a hot air oven at $60^{\circ} \mathrm{C}$ for three days, and dry biomass was observed.

\section{Transcriptome Analysis}

Uniformly germinated WT and OsJAZ9 overexpressing line OE (OE10) seedlings were transferred to $4.08 \mu \mathrm{M}$ and $510 \mu \mathrm{M}$ concentrations of $\mathrm{K}_{2} \mathrm{SO}_{4}$ for 15 days along with other nutrients as per Yoshida liquid media. After that, total RNA was isolated with Qiagen RNeasy Mini Kit from the whole seedling according to the manufacturer's protocol. RNA integrity was analyzed using Bioanalyzer (2100 Agilent technologies), and samples with RIN value $\geq 7.5$ proceeded for library preparation. Transcriptome sequencing was done using the Illumina Hiseq 2500/4000 platform with two independent biological replicates for each WT and OsJAZ9 OE10. Adapter sequences and low-quality bases were trimmed using AdapterRemoval v2 (version 2.2.0). Further, rRNA sequences were removed by aligning reads with silva database. Finally, processed reads were aligned with the MSU7 genome (http://rice.plantbiology.msu.edu/pub/data/Eukaryotic_Projects/o_sativa/annotation_dbs/pseu domolecules/version_7.0/all.dir//) using the STAR program (Version 2.5.3a). Differentially expressed genes were identified as described (Bandyopadhyay et al., 2017).

\section{JA quantification}


For JA and JA-Ile quantification, uniformly germinated WT and OsJAZ9 overexpression (OE10) seedlings were transferred to $4.08 \mu \mathrm{M}$ and $510 \mu \mathrm{M}$ concentrations of $\mathrm{K}_{2} \mathrm{SO}_{4}$ in Yoshida liquid media for 15 days. Whole seedlings were harvested, freezed and stored at $-80^{\circ} \mathrm{C}$ until used. For JAs and JA-Ile estimation, 250mg of total seedlings was homogenised and extraction was done with $1.5 \mathrm{ml}$ methanol containing $60 \mathrm{ng}$ of 9,10-d2-9,10-dihydrojasmonic acid and 15 ng of JA-[13C6]Ile conjugate as internal standards. The homogenate was for $30 \mathrm{~min}$ using vortex followed by spin at $14000 \mathrm{rpm}$ for $20 \mathrm{~min}$ at $4^{\circ} \mathrm{C}$. A second extraction was again done with $0.5 \mathrm{ml}$ methanol and the supernatant was pooled. The combined supernatant was evaporated in a SpeedVac and re-dissolved in $0.5 \mathrm{ml}$ methanol. JA and JA-Ile concentrations were quantified in at least four replicates, as described in Vadassery et al. (2012). JAs and JAIle were quantified on Exion LC coupled to Triple Quad 6500+ (Sciex). JAs and JA-Ile were quantified relative to the signal of their corresponding internal standard.

\section{RESULTS}

\section{$\mathrm{K}$ deficiency promotes $\mathrm{JA}$ signaling in rice}

Our low potassium dose-response experiment showed drastic root and shoot growth inhibition of rice seedlings (Fig. S1). To underpin the underlying genes responsible for root and shoot growth inhibition, we performed RNA-seq analysis and compared the gene expression in WT plants grown under $\mathrm{K}$ sufficient and deficient conditions. Interestingly, we found induction of several JA biosynthetic genes like $O s L O X 8, O s A C X 2$, OsOPR10, OsJAO1 etc. (Table S2). To validate this observation, we performed temporal expression profiling of selected JAassociated genes under K deficiency using qRT-PCR. This analysis confirmed the induced expression of $O s C O I 1 b$ and $O s M Y C 2$ on $\mathrm{K}$ deficiency suggesting elevated JA signalling. Moreover, we also found increased expression of OsAOS1, OsAOS2 and OsJAR1 suggesting increased JA biosynthesis under K deficiency (Fig. S2). These results indicate a higher JA level and concomitantly induced JA signalling during the initial phase of $\mathrm{K}$ deficiency in rice.

In our previous study, we had reported highest downregulation of OSJAZ9 especially at early time points under low K conditions (Singh et al., 2015). To extrapolate this result, we performed an early time point expression analysis of OSJAZ9 under $\mathrm{K}$ deficiency and found down-regulation of OSJAZ9 transcripts at initial time point (Fig. S2A). Our pOsJAZ9:GUS transcriptional reporter lines also showed transcriptional down-regulation of OsJAZ9 in a temporal fashion (Fig. 1). On the basis of these results, we hypothesized that $\mathrm{K}$ deficiency 
provokes increased JA signalling and biosynthesis, and OsJAZ9 may be involved here. Moreover, we also found OsJAZ9 as a nuclear-localized JAZ protein (Fig. S3).

JA signalling alters both RNA and protein levels of JAZ repressors. Therefore, to study the effect of OsJAZ9 on K deficiency, we raised dual purpose overexpression lines of OsJAZ9 (CaMV35S:OSJAZ9_GUS). In addition to overexpression of OSJAZ9, these lines also serve the function of a translational reporter. Expectedly, MeJA treatment promoted the degradation of complete JAZ9-GUS signal however, GUS signal was intact in the roots of the OsJAZ9-GUS reporter without C-terminal Jas domain implying that OsJAZ9 is degraded in the presence of JA through Jas domain (Fig. 2B). Further, MeJA supplemented with MG-132 (proteasome inhibitor) was unable to degrade OsJAZ9_GUS signal, which confirms that JAs degrade OsJAZ9 via proteasomal pathway (Fig. 2B).

Therefore, we utilized these two lines to investigate JA response during $\mathrm{K}$ deficiency considering OsJAZ9_GUS stability as a proxy for JA signalling response. Interestingly, initial exposure of $\mathrm{K}$ deficiency (up to $3 \mathrm{~h}$ ) showed a rapid decrease in OsJAZ9_GUS signals. However, after 6h, OsJAZ9_GUS signal was stabilized indicating early induction of JA signaling under $\mathrm{K}$ deficiency (Fig. 2C). However, on prolonged $\mathrm{K}$ deficiency exposure, OsJAZ9_GUS signal remains stabilized until $12 \mathrm{~h}$ and starts decreasing after that. Moreover, after 15 days of K deficiency, OsJAZ9_GUS signal again disappeared completely (Fig. S4B). This revealed a kind of rhythmic stability of OsJAZ9 levels during the early phase of $\mathrm{K}$ deficiency. Expectedly, OsJAZ9AC_GUS line showed consistent GUS signal throughout the duration of $\mathrm{K}$ deficiency (Fig. 2C and S4B). These results confirmed that JA levels are indeed enhanced during $\mathrm{K}$ deficiency.

\section{Overexpression of $O s J A Z 9$ enhances $\mathrm{K}$ deficiency tolerance in rice}

To characterize the functional roles of OsJAZ9 under K deficiency, we analysed homozygous lines of OsJAZ9 overexpression (OE2 and 10) and RNAi (RN12 and 14). The overexpression lines had significant upregulation while RNAi lines showed around 98\% reduction in the OsJAZ9 expression as compared to WT (Fig. S5). We next tested the growth performance of WT and transgenic lines grown in $+\mathrm{K}$ (control) and $-\mathrm{K}$ ( $\mathrm{K}$ deficient) media for fifteen days. Phenotypic analysis revealed that $\mathrm{K}$ deficiency inflicted lesser plant growth inhibition in OsJAZ9 overexpressing lines as compared to WT while RNAi lines showed no significant difference in the shoot biomass (Fig. S6; S7). 
Furthermore, we performed another experiment including OsJAZ9 OE, RNAi and WT seedlings in $\mathrm{K}$ sufficient media for 10 days followed by $\mathrm{K}$ deficiency for the next 15 days and thereafter recovery in $\mathrm{K}$ sufficient media for next 5 days. OSJAZ9 OE plants were preforming significantly better than WT and RNAi lines by accumulating more root and shoot dry biomass under both $\mathrm{K}$ deficiency and $\mathrm{K}$ recovery conditions. (Fig. 3). Even, the root numbers (seminal and crown root) in OsJAZ9 OE plants were also significantly higher than WT and RNAi lines under $\mathrm{K}$ deficiency and recovery conditions (Fig. 3). Interestingly, we also observed a higher $\mathrm{Na}^{+} / \mathrm{K}^{+}$ratio in OE plants than both WT and RNAi lines during $\mathrm{K}$ deficient conditions (Fig. 3). Further, during recovery conditions this $\mathrm{Na}^{+} / \mathrm{K}^{+}$in $\mathrm{OE}$ plants was found significantly lower than both WT and RNAi lines. Moreover, we observed higher K content in OE plants during recovery conditions which remained non-significant during $\mathrm{K}$ deficient conditions. Since, during $\mathrm{K}$ deficient conditions there was no external $\mathrm{K}$ available for uptake and $\mathrm{OE}$ plants absorb $\mathrm{Na}^{+}$which is the structural mimic of $\mathrm{K}^{+}$which resulted into higher $\mathrm{Na}^{+} / \mathrm{K}^{+}$in $\mathrm{OE}$ lines.

In order to investigate better $\mathrm{K}$ uptake in OsJAZ9 overexpression plants, we analysed the expression pattern of known $\mathrm{K}^{+}$transporters under $\mathrm{K}$ sufficient, deficient and recovery conditions. Expression profiling (including OsHKT2;1, OsHAK5 and OsHAK1) showed induced expression of these $\mathrm{K}^{+}$transporting genes under $\mathrm{K}$ deficient and recovery conditions (Fig. S8A). Moreover, we also found higher expression of OsHKT2; 1 and OsHAK1 in OsJAZ9 OE lines, especially after $12 \mathrm{~h}$ of $\mathrm{K}$ recovery (Fig. S8B). The higher expression of $\mathrm{K}$ transporters well supports the better $\mathrm{K}$ uptake in OsJAZ9 OE plants during $\mathrm{K}$ recovery conditions.

\section{Transcriptome profiling revealed enhanced JA biosynthesis in OsJAZ9 OE lines in rice}

Overexpression of several $J A Z$ repressor encoding genes under the constitutive promoter has resulted in partial JA insensitivity in Arabidopsis and rice (Thines et al., 2007; Yamada et al., 2012). To understand the JA sensitivity level of the JAZ9 transgenics, we analyzed root growth inhibition of OsJAZ9 OE and RNAi lines upon MeJA treatment. Consistent with previous studies, we also observed a partial JA insensitive phenotype in OsJAZ9 OE lines (Fig. 4). We found $\sim 36 \%$ root growth inhibition in RNAi line, whereas only $11 \%$ reduction in overexpressing lines (OE10) as compared to WT (30\% root length reduction) upon exogenous MeJA treatment. This experiment also suggests that OsJAZ9 OE may have higher level of bioactive JA. 
To uncover the global transcriptional response of $O S J A Z 9$ overexpression under $\mathrm{K}$ deficiency, we performed RNA-seq analysis of WT and OsJAZ9 OE (OE10) seedlings raised under normal and $\mathrm{K}$ deficient conditions for 15 days. The comparative $+\mathrm{K}$ vs. $-\mathrm{K}$ analysis revealed a total of 5173 and 5505 genes were differentially expressed $(\mathrm{p}<0.05, \mathrm{q}<0.05)$ in WT and OsJAZ9 OE line, respectively, during K deficiency. Out of these 2433 and 2670, genes were upregulated, and 2740 and 2835 genes were downregulated, respectively, in WT and OsJAZ9 OE line (Fig. 5 A-B).

We further analyzed the expression of all known JA biosynthesis and signaling genes in our transcriptome data. Out of 17 JA-biosynthesis related genes detected in the transcriptome, seven were showing higher expression in OE plants as compared to WT during K deficiency considering their respective controls while four genes were downregulated (Table S3). We also validated our RNA-seq results by qRT-PCR analysis and most of the selected genes showed very similar expression pattern (Fig. S9).

We found up-regulation of OsAOS2, OsLOX5, OsLOX7, OsLOX8, OsACX2, OsOPR5, and OSJAO1 which play key roles in JA biosynthesis (or homeostasis) and higher expression of key JA signaling marker genes like OSVSP2 in OsJAZ9 OE plants than WT plants subjected to $\mathrm{K}$ deficiency (Table S3). This higher transcript abundance of JA biosynthesis, signaling and marker genes confirmed the elevated JA response in OSJAZ9 OE plants during K deficiency as compared to the WT. Further analysis revealed that out of 13 known K transporters, 7 were having higher transcript abundance in OE line as compared to WT under K deficiency, while 6 transporters were downregulated (Table S4). Among the lesser expressed ones, OsHKT27 encodes a K efflux transporter, OsHKT2;2 and OsHKT1;5 were described as more efficient in $\mathrm{Na}$ transport than K (Kadar et al., 2006) while OsK1.1 (OsAKT1) functions at the millimolar range of external K (Ahmad et al., 2016). We found OsHAK10, OsHAK24, OsK3.1, OsK5.2, OsHKT1;1, OsHKT1;4, OsSOS1 and OsGORK genes having higher abundance in OE line as compared to WT in K deficient conditions and thus supports the case for higher $\mathrm{K}$ uptake in OsJAZ9 overexpressing plants (Table S4).

\section{OsJAZ9 modulates root system architecture}

As overexpression of $O s J A Z 9$ resulted in higher $\mathrm{K}$ levels in transgenic plants, we analyzed root traits that could contribute to $\mathrm{K}$ uptake in addition to the $\mathrm{K}$ transporter activity. Interestingly, OSJAZ9 OE plants showed shorter seminal roots while RNAi plants have longer seminal roots both in $\mathrm{K}$ deficient and sufficient conditions (Fig 6). However, OsJAZ9 OE lines had longer 
lateral roots in both low and optimal K conditions (Fig. 6C). In WT background, we also found higher expression of OSJAZ9 in elongation and maturation zones as compared to the root tip of seminal roots. On comparison, qRT-PCR analysis showed higher OsJAZ9 expression in lateral roots than primary root suggesting a role for OsJAZ9 in regulating lateral root growth (Fig. S10).

Previously, low doses of exogenous JA were reported to enhance the lateral root length in Arabidopsis and rice, while at higher doses, JA inhibits lateral elongation (Raya-Gonzalez et al., 2012; Hsu and Kao, 2011). We speculated that this slow growth of seminal roots along with enhanced lateral elongation could be either due to higher levels of intrinsic JA or enhanced JA signaling in OsJAZ9 OE lines. To test these possibilities, we analyzed the effect of exogenous MeJA application on RSA (Root System Architecture) of WT plants grown in control $(510 \mu \mathrm{M}$ $\mathrm{K}_{2} \mathrm{SO}_{4}$ ) and $\mathrm{K}$ deficient ( $4.08 \mu \mathrm{M} \mathrm{K}_{2} \mathrm{SO}_{4}$ ) conditions for 15 days under varying concentrations of MeJA. Interestingly, we observed increased lateral root length at lower concentrations of MeJA $(0.001$ and $0.01 \mu \mathrm{M})$ while the higher level of MeJA $(1 \mu \mathrm{M})$ inhibited the lateral root elongation under both control and $\mathrm{K}$ deficient conditions (Fig. S11; S12). In agreement with Raya-Gonzalez et al. (2012) and Hsu and Kao (2011), we also observed increased lateral root numbers at lower concentrations of exogenous MeJA (Fig. S11C; S12C). Again, longer laterals roots were overrepresented in lower MeJA treatment as compared to no MeJA or higher MeJA concentrations (Fig. S11D; S12D). These observations also validated the hypothesis that OSJAZ9 OE plants are experiencing higher JA level which is also reflected in our transcriptome data.

As we found OSJAZ9 overexpression transgenics modulate RSA, we were interested to understand tissue specific expression of OSJAZ9 in various organ of roots. For this, we generated stable transcriptional reporter lines of OsJAZ9 with GUS ( $p O s J A Z 9: G U S$ ). GUS staining of roots of $p O S J A Z 9: G U S$ reporter line showed expression of OSJAZ9 only confined in lateral roots (Fig. 1). Remarkably, OsJAZ9 promoter:GUS signal was faded away when the reporter lines were transferred to a low $\mathrm{K}$ regime and completely vanished after 15 days of low $\mathrm{K}$ treatment in lateral roots (Fig. 1). This lateral root-specific expression pattern confirms the role of $O S J A Z 9$ as evidenced in regulating lateral root elongation. qRT-PCR analysis further confirmed the higher expression of OsJAZ9 in lateral roots (LR) than the rest of the root (primary root, PR). This validated the qRT-PCR result of OsJAZ9 expression in other root organs. 
As overexpression lines of $O s J A Z 9$ contained a large GUS tag, we suspected that our findings may be influenced by under/over-estimation of OsJAZ9 functions in $\mathrm{K}$ deficiency. To exclude any such possibility or observation bias, we also raised overexpression lines of 'untagged OSJAZ9' under ZmUbil promoter. We screened these transgenic lines and reanalysed their phenotypes under $\mathrm{K}$ sufficient and deficient conditions (Fig. S13). Notably, the new overexpression lines also behaved similar to the studied translational reporter lines under $\mathrm{K}$ deficiency (Fig. S14; S15). This reconfirmed our conclusions about roles of OsJAZ9 under K deficiency.

\section{Overexpression of $O s J A Z 9$ enhances in-vivo JA-Ile levels under $\mathrm{K}$ deficiency}

The observed phenotypes of OSJAZ9 OE lines so far suggest invariably heightened JA response which might be contributed by either increased intrinsic JA levels or the increased JA signaling in OsJAZ9 OE lines as compared to the WT. To test whether there was indeed higher JA content in OsJAZ9 OE plants or these plants are only experiencing elevated JA signaling; we analyzed the levels of JA and JA-Ile levels in WT and OsJAZ9 overexpression (OE10) seedlings raised under $\mathrm{K}$ deficient and sufficient conditions as compared to their respective controls. We found slightly higher JA levels in OE plants as compared to WT plants under both K sufficient and $\mathrm{K}$ deficient conditions (Fig. S16). To our surprise, we observed no change in JA-Ile content in OE plants as compared to WT under K sufficient conditions. However, JA-Ile content was significantly increased in OE plants as compared to WT plants in response to $\mathrm{K}$ deficiency. The induction was four fold in WT plants while it was around 8 fold in OE plants under K deficiency (Fig. 7). Based on this evidence, we conclude that overexpression of OsJAZ9 results in enhanced bioactive JA levels during $\mathrm{K}$ deficiency, which is also reflected in the transcriptome data.

\section{DISCUSSION}

Potassium is one of the essential minerals for plant growth. Despite its high concentration in soil, it remains mostly unavailable for root uptake and utilization. Therefore, its deficiency in soil remains one of the major constraints for crop productivity. As $\mathrm{K}^{+}$being a structural mimic of $\mathrm{Na}^{+}$, its deficiency also results in high $\mathrm{Na}^{+}$uptake by the plant and causes salinity stress. Therefore, $\mathrm{K}$ availability in the soil also influences the level of salt stress experienced by the plant. In contrast to Phosphorus (P) deficiency responses, where enzymes like purple acid phosphatase can elevate the P availability in soil (Mehra et al., 2017), there is no such enzyme known in rice to hydrolyze locked-up $\mathrm{K}$ in the soil. Therefore, strategies targeting exploiting 
of $\mathrm{K}$ transporters and reshaping of root architecture are more suited for enhancing K-uptakeefficiency in rice.

While JAs are well-known for controlling plant defence against fungal and insect attacks (Peng et al., 2012; Wasternack and Hause, 2013), emerging evidences have established their role in diverse plant processes like flower development, reproduction, and response to abiotic and biotic stresses (Cai et al., 2014; Kazan, 2015; Nguyen et al., 2019). JAs are also known for inhibiting root growth, and recently intricate details of this response were revealed (Wasternack and Hause, 2013; Wasternack and Song, 2017). Root growth inhibition during K deficiency mimics the root growth inhibition phenotype observed on exogenous JA treatment in various plants (Staswick et al., 1992; Gruber et al., 2013; Cai et al., 2014). Recently, Li et al. (2017) and Troufflard et al. (2010) have found enhanced JA levels during K deficiency in Arabidopsis, rice, and wheat. JAZ repressors are pivotal to JA signaling, and their genetic manipulation can lead to many altered plant processes (Wasternack and Song, 2017). We have reported previously that $O S J A Z$ genes encoding JA signaling repressors are transcriptionally responsive to $\mathrm{K}$ deficiency (Singh et al., 2015). However, it was not known if JAZs can also regulate $\mathrm{K}$ deficiency responses. Here, we found that OsJAZ9 expression and stability form a rhythmic/wavy pattern indicating a faster early response and a slower late response towards $\mathrm{K}$ deficiency (Fig. 1, S2 and S4). Similar wavy patterns were also observed for $J A Z$ genes expression in Arabidopsis upon exogenous JA treatment (Hickman et al., 2018). These observations strengthen our hypothesis that $\mathrm{K}$ deficiency response involves enhanced JA response.

Here using comprehensive transgenics, physiological and morphological approaches, we showed that OsJAZ9 is a crucial component of JA signaling for $\mathrm{K}$ deficiency responses. OsJAZ9 OE lines performed better in terms of root and shoot growth under $\mathrm{K}$ deficiency, while RNAi lines performed poorly. We also noticed that OSJAZ9 overexpression plants are showing both better K-uptake-efficiency (higher $\mathrm{K}$ levels in OsJAZ9 OE plants under recovery as compared to WT) and K-use-efficiency (OsJAZ9 OE plants were able to produce more biomass during $\mathrm{K}$ deficient conditions). In addition, higher $\mathrm{Na}^{+} / \mathrm{K}^{+}$found in $\mathrm{OE}$ lines during $\mathrm{K}$ deficient conditions was reflecting increased $\mathrm{Na}^{+}$uptake while $\mathrm{K}^{+}$contents were less affected. This could be explained well as $\mathrm{Na}^{+}$and $\mathrm{K}^{+}$are the structural mimics of each other and $\mathrm{Na}^{+}$can be taken up more when $\mathrm{K}^{+}$is not available to the plants, which can result in a higher $\mathrm{Na}^{+} / \mathrm{K}^{+}$ratio under extremely $\mathrm{K}$ deficient conditions $(0 \mu \mathrm{M} \mathrm{K})$. This becomes clearer when the $\mathrm{K}$ starved plants were recovered with $10 \mu \mathrm{M} \mathrm{K}_{2} \mathrm{SO}_{4}$ and $\mathrm{Na}^{+} / \mathrm{K}^{+}$goes down in $\mathrm{OE}$ lines, but it remains high in 
RNAi lines. This decrease of $\mathrm{Na}^{+} / \mathrm{K}^{+}$ratio was more due to the efficient $\mathrm{K}^{+}$uptake rather $\mathrm{Na}^{+}$ uptake by $\mathrm{OE}$ lines as indicated by the $\mathrm{K}^{+}$content per plant. These results suggested that OSJAZ9 has improved $\mathrm{K}^{+}$over $\mathrm{Na}^{+}$uptake during low $\mathrm{K}^{+}$availability. The elevated $\mathrm{K}^{+}$uptake of OsJAZ9 OE lines was well supported by the higher transcript abundance of $\mathrm{K}$ transporters, OsHAK1 and OsHKT2;1.

Interestingly, we did not find $O s N H X 1$ induction under K deficiency while its transcripts were induced during $\mathrm{NaCl}$ excess stress (Wu et al., 2015). Thus its non-responsive nature towards $\mathrm{K}$ deficiency shows that $O s N H X 1$ is very specific for its exclusive expression in $\mathrm{NaCl}$ stress. This expression behaviour demarcates the thin boundary between salt and K deficiency stress. A higher magnitude of transcriptome changes in OsJAZ9 OE lines further also showed greater responsiveness towards K deficiency than WT. Particularly, enhanced expression of JA biosynthesis and marker genes like $O s A O S 2$, OsLOX5/7/8, OsJAO1 and OsVSP2, respectively, confirms the higher JA levels in OsJAZ9 OE plants during K deficiency.

The enhanced JA response on overexpression of a repressor is surprising; however, similar observations on activated JA signaling were also made on $J A Z 7$ overexpression in Arabidopsis (Thatcher et al., 2016). Therefore, the intricate contribution of OsJAZ9 with positively and negatively interacting partners of the JA signaling machinery needs to be elucidated. How the overexpression of a JAZ repressor (OsJAZ9) influence the increased JA biosynthesis is not clear. Does JAZ9 also activate some component of JA biosynthesis machinery would answer this question. If overexpression of $O s J A Z 9$ suppresses the downstream JA signalling by supressing the OsMYC2 then why JA response is elevated in OsJAZ9 overexpressing transgenic seems counterintuitive. In fact we observed some JA signalling genes being upregulated in OSJAZ9 OE plants. This implies that JA biosynthesis machinery is directly or indirectly influenced by OsJAZ9. Further pulldown assay of OsJAZ9 interactome will reveal several aspects of its action.

In addition to enhanced $\mathrm{K}$ transporters activity, longer laterals might have also contributed to improved K uptake in OsJAZ9 OE plants. It appears that seminal root length was inhibited in OE lines, but an increase in laterals length might have increased root surface area for better $\mathrm{K}$ acquisition. This also suggest that plant have different level of JA sensitivity for lateral elongation and seminal elongation. It would be fascinating to reveal how same level of JA act differently to augment lateral elongation and seminal growth suppression. We cannot rule out the possibility of having different signal amplification (JA response) in lateral and seminal 
roots. Our transcriptional reporter lines show that $O S J A Z 9$ is preferentially expressed in elongating lateral roots than seminal root tips. Therefore, relatively (seminal vs lateral) a small dose of external JA application might have distinct effect on laterals than seminal roots. Previously, exogenous MeJA has resulted in the enhanced lateral root length and density in Arabidopsis (Raya-González et al., 2012; Hsu and Kao, 2011). Our observations of increased lateral root elongation by low concentrations of MeJA, confirm the growth-promoting nature of JA at a low level, and parallel the longer lateral phenotype of OsJAZ9 OE lines. However, it may hold true only at lower levels of JA as higher exogenous JA is growth inhibitory irrespective of the stress.

At morphological levels, JA treatment is known to inhibit the primary root growth in plants (Staswick et al., 1992; Cai et al., 2014). Consistent with these studies, we also found OsJAZ9 OE plants having shorter roots than WT, mimicking the canonical JA overproduced or enhanced JA signaling response. This phenotype is very appealing as most of the other $J A Z$ repressors overexpressed transgenics in Arabidopsis (JAZ1, JAZ2, JAZ3, JAZ5, JAZ7, and $J A Z 9)$ and rice $(J A Z 1)$ reported earlier, either promoted growth or showed no change (Chini et al., 2007; Hakata et al., 2017; Yang et al., 2012). Like other JAZs, root growth inhibition assay revealed reduced JA sensitive behavior of OsJAZ9 OE lines while knockdown lines were JA hypersensitive.

The induction of JA biosynthetic genes and the phenotypes mimicking the JA treatment made us analyze the JA and JA-Ile content in transgenic lines. The slightly enhanced JA and highly induced JA-Ile accumulation in OsJAZ9 transgenic lines explain higher JA response in OE lines during $\mathrm{K}$ deficiency. Mostly, overexpression of Arabidopsis JAZs inhibits JA induced growth suppression while the loss of function mutants of AtJAZs exhibited hypersensitivity to JA (reviewed in Wasternack and Song, 2017). In agreement with Wu et al. (2015), we also found JA hypersensitive response in OsJAZ9 knockdown lines. However, elevated JA-Ile levels and signaling in $O S J A Z 9 \mathrm{OE}$ lines are very intriguing.

We suspect here a negative feedback regulation of JA homeostasis wherein constitutive overexpression of a JAZ repressor hyperactivated JA signaling (Chini et al., 2007; Thatcher et al., 2016). JAZs activities are tightly controlled at tissue levels. By constitutively overexpressing a JAZ repressor, we may have disturbed that fine-tuned JA homeostasis resulting in enhanced JA levels which in-turn lead to enhanced JA response in OsJAZ9 overexpression plants as also reported earlier in Arabidopsis (Thatcher et al., 2016). 
486 Noticeably, JA levels were also enhanced in knockdown lines of AtJAZ1 while it remained unchanged in the knockdown lines of NaJAZh (Li et al., 2017; Oh et al., 2012).

A closer look at the transcriptome data revealed the suppression of all $J A Z$ repressors under both normal and low $\mathrm{K}$ conditions in OsJAZ9 overexpression plants (Table S5). This makes OsMYC2 free from the clutch of JAZ repressors, which leads to enhanced JA response. This elevated JA response can shape the overall phenotype and its effect on root growth. The suppression of other $J A Z s$ can underscore the effect of $O s J A Z 9$ overexpression. How does low $\mathrm{K}$ level invokes JA biosynthesis will pave the way for a broader understanding of low $\mathrm{K}$ perception and its close association with JA machinery. However, our multifaceted evidence supports the role of $O S J A Z 9$ as an important element regulating JA-biosynthesis and response and thus influencing root system architecture and $\mathrm{K}$ homeostasis.

\section{ACKNOWLEDGMENTS}

A.P.S., P.M., and B.K.P. acknowledge the research fellowship by UGC, CSIR, and DBT, respectively. We thank Dr. Vijayata Singh, CSSRI, Karnal, for help in K and Na estimation. J.G. acknowledges a grant from the INSA-young scientist project. We acknowledge the NIPGR metabolomics facility.

\section{AUTHORS CONTRIBUTIONS}

503

504

505

A.P.S., B.K.P., and P.M. conducted experiments and analyzed data. J.G. designed the project, supervised experiments. A.P.S., B.K.P., P.M., T.H., and J.G. wrote the manuscript.

\section{References}

Ahmad I, Mian A, Maathuis FJM (2016) Overexpression of the rice AKT1 potassium channel affects potassium nutrition and rice drought tolerance. Journal of Experimental Botany 67:2689-2698.

Armengaud P, Breitling R, Amtmann, A (2004) The potassium-dependent transcriptome of Arabidopsis reveals a prominent role of jasmonic acid in nutrient signaling. Plant Physiology 136:2556-2576.

Bandyopadhyay T, Mehra P, Hairat S, Giri J (2017) Morpho-physiological and transcriptome profiling reveal novel zinc deficiency-responsive genes in rice. Functional \& Integrative Genomics 17:565-581. 
Cai Q, Yuan Z, Chen M, Yin C, Luo Z, Zhao X, Liang W, Hu J, Zhang D (2014) Jasmonic acid regulates spikelet development in rice. Nature Communications 5:3476.

Chini A, Fonseca S, Fernández G, et al. (2007) The JAZ family of repressors is the missing link in jasmonate signalling. Nature 448:666-671.

Egilla JN, Davies FT, Drew MC (2001) Effect of potassium on drought resistance of hibiscus rosa-sinensis cv. Leprechaun: plant growth, leaf macro- and micronutrient content and root longevity. Plant and Soil 229:213-224.

Elumalai RP, Nagpal P, Reed JW (2002) A mutation in the Arabidopsis kt2/kup2 potassium transporter gene affects shoot cell expansion. Plant Cell 14:119-131.

Feys BJF, Benedetti CE, Penfold CN, Turner JG (1994) Arabidopsis mutants selected for resistance to the phytotoxin coronatine are male sterile, insensitive to methyl jasmonate, and resistant to a bacterial pathogen. Plant Cell 6:751-759.

Fu J, Wu H, Ma S, Xiang D, Liu R, Xiong L (2017) OsJAZ1 Attenuates Drought Resistance by Regulating JA and ABA Signaling in Rice. Front Plant Sci. 8:2108.

Gruber BD, Giehl RFH, Friedel S, von Wirén N (2013) Plasticity of the Arabidopsis root system under nutrient deficiencies. Plant Physiology 163:161-179.

Hakata M, Muramatsu M, Nakamura H, et al. (2017) Overexpression of TIFY genes promotes plant growth in rice through jasmonate signaling. Bioscience, Biotechnology and Biochemistry 81:906-913.

Hardter R. (2002) Potassium and biotic stress of plants. In: Feed the soil to feed the people. The role of potash in sustainable agriculture (ed Johnston A.E.), pp. 345-362. International Potash Institute Basel, Switzerland.

Heitz T, Smirnova E, Widemann E, Aubert Y, Pinot F, Ménard R (2016) The Rise and Fall of Jasmonate Biological Activities. Subcell Biochem. 86:405-26.

Hickman R, Van Verk MC, Van Dijken AJH, et al. (2017). Architecture and Dynamics of the Jasmonic Acid Gene Regulatory Network. The Plant Cell 29:2086-2105.

Hsu YY, Kao CH (2011) Nitric oxide is involved in methyl jasmonate-induced lateral root formation in rice. Crop Environment and Bioinformatics 8:160-7. 
Kader MA, Seidel T, Golldack D, Lindberg S (2006) Expressions of OsHKT1, OsHKT2, and OsVHA are differentially regulated under $\mathrm{NaCl}$ stress in salt-sensitive and salt-tolerant rice (Oryza sativa L.) cultivars. Journal of Experimental Botany 57:4257-4268.

Kant S, Kafkafi U (2002) Potassium and abiotic stresses in plants. In: Potassium for sustainable crop production. Potash Institute of India (eds Pasricha NS, Bansal SK), pp. 233-251. Gurgaon.

Kaya C, Kirnak H, Higgs D (2006) Enhancement of growth and normal growth parameters by foliar application of potassium and phosphorus in tomato cultivars grown at high $(\mathrm{NaCl})$ salinity. Journal of Plant Nutrition 24:357-367.

Kazan K (2015) Diverse roles of jasmonates and ethylene in abiotic stress tolerance. Trends in Plant Science 20:219-229.

Kobayashi T, Itai RN, Senoura T, Oikawa T, Ishimaru Y, Ueda M, Nakanishi H, Nishizawa NK (2016) Jasmonate signaling is activated in the very early stages of iron deficiency responses in rice roots. Plant Molecular Biology 91:533-547.

Leigh RA, Wyn Jones RG (1984). A hypothesis relating critical potassium concentrations for growth to the distribution and functions of this ion in the plant cell. New Phytologist 97:1-13.

Li G, Wu Y, Liu G et al (2017) Large-scale proteomics combined with transgenic experiments demonstrates an important role of jasmonic acid in potassium deficiency response in wheat and rice. Molecular and Cellular Proteomics 16:1889-1905.

Ma TL, Wu WH, Wang Y (2012) Transcriptome analysis of rice root responses to potassium deficiency. BMC Plant Biology 12:161.

Mann DG, LaFayette PR, Abercrombie LL et al (2012) Gateway-compatible vectors for highthroughput gene functional analysis in switchgrass (Panicum virgatum L.) and other monocot species. Plant Biotechnology Journal 10:226-236.

Mehra P, Giri J (2016) Rice and chickpea GDPDs are preferentially influenced by low phosphate and CaGDPD1 encodes an active glycerophosphodiester phosphodiesterase enzyme. Plant Cell Report 35:1699-1717. 
Mehra P, Pandey BK, Giri J (2017) Improvement in phosphate acquisition and utilization by a secretory purple acid phosphatase (OsPAP21b) in rice. Plant Biotechnology Journal 15:1054-1067.

Mengel K, Viro M (1974) Effect of Potassium Supply on the Transport of Photosynthates to the Fruits of Tomatoes (Lycopersicon esculentum). Physiologia Plantarum 30:295-300.

Murashige T, Skoog F (1962) A Revised Medium for Rapid Growth and Bio Assays with Tobacco Tissue Cultures. Physiologia Plantarum 15:473-497.

Nguyen HT, To HTM, Lebrun M, Bellafiore S, Champion A (2019) Jasmonates- - the master regulator of rice development, adaptation and defense. Plants 2019, 8:339.

Oh Y, Baldwin IT, GÃlis I (2012) NaJAZh Regulates a Subset of Defense Responses against Herbivores and Spontaneous Leaf Necrosis in Nicotiana attenuata Plants. Plant Physiology 159:769-788.

Pandey BK, Mehra P, Verma L, Bhadouria J, Giri J (2017) OsHAD1, a haloacid dehalogenaselike APase enhances phosphate accumulation. Plant Physiology 174:2316-2332.

Qi Z, Spalding EP (2004) Protection of plasma membrane $\mathrm{K}^{+}$transport by the salt overly sensitive1 $\mathrm{Na}^{+} / \mathrm{H}^{+}$antiporter during salinity stress. Plant Physiology 136:2548-2555.

Raya-González J, Pelagio-Flores R, López-Bucio J (2012) The jasmonate receptor COI1 plays a role in jasmonate-induced lateral root formation and lateral root positioning in Arabidopsis thaliana. Journal of Plant Physiology 169:1348-1358.

Rich CI, Black WR (1964) Potassium exchange as affected by cation size, $\mathrm{pH}$ and mineral structure. Soil Science 97:384-390.

Rich CI (1964) Effect of cation size and ph on potassium exchange in nason soil. Soil Science 98:100-106.

Riemann M, Dhakarey R, Hazman M, Miro B, Kohli A, Nick P (2015) Exploring jasmonates in the hormonal network of drought and salinity responses. Frontiers in Plant Science 6:1077.

Sarwar M (2012) Effects of potassium fertilization on population build up of rice stem borers (lepidopteron pests) and rice (Oryza sativa 1.) yield. Journal of Cereals and Oilseeds 3:6-9. 
600

601

602

603

604

605

606

607

608

609

610

611

612

613

614

615

616

617

618

619

620

621

622

623

624

625

626

627

628

Sawhney BL, Zelitch I (1969) Direct determination of potassium ion accumulation in guard cells in relation to stomatal opening in light. Plant Physiology 44:1350-1354.

Shankar A, Singh A, Kanwar P, Srivastava AK, Pandey A, Suprasanna P, Kapoor S, Pandey GK (2013) Gene expression analysis of rice seedling under potassium deprivation reveals major changes in metabolism and signaling components. PLoS One 8:e70321.

Sheard LB, Tan X, Mao H et al (2010) Jasmonate perception by inositol-phosphate-potentiated COI1-JAZ co-receptor. Nature 468:400-405.

Singh AP, Pandey BK, Deveshwar P, Narnoliya L, Parida SK, Giri J (2015) JAZ Repressors: Potential involvement in nutrients deficiency response in rice and chickpea. Frontiers in Plant Science 6:975.

Sparks DL (1987) Potassium dynamics in soils. In: Advances in soil science (ed Stewart B.A.), pp. 1-63. Springer, New York.

Staswick PE, Su W, Howell SH (1992). Methyl jasmonate inhibition of root growth and induction of a leaf protein are decreased in an Arabidopsis thaliana mutant. Proceedings of the National Academy of Sciences 89:6837-6840.

Takehisa H, Sato Y, Antonio BA, Nagamura Y (2013) Global transcriptome profile of rice root in response to essential macronutrient deficiency. Plant Signaling and Behavior 8:e24409.

Terry N, Ulrich A (1973) Effects of potassium deficiency on the photosynthesis and respiration of leaves of sugar beet. Plant Physiology 51:783-786.

Tester M, Blatt MR (1989) Direct measurement of k+ channels in thylakoid membranes by incorporation of vesicles into planar lipid bilayers. Plant Physiology 91:249-252.

Thatcher LF, Cevik V, Grant M, Zhai B, Jones JD, Manners JM, Kazan K (2016) Characterization of a JAZ7 activation-tagged Arabidopsis mutant with increased susceptibility to the fungal pathogen Fusarium oxysporum. Journal of Experimental Botany 67:2367-2386.

Thines B, Katsir L, Melotto M, Niu Y, Mandaokar A, Liu G, Nomura K, He SY, Howe GA, Browse J (2007) JAZ repressor proteins are targets of the SCF(COI1) complex during jasmonate signalling. Nature 448:661-665. 
Troufflard S, Mullen W, Larson TR, Graham IA, Crozier A, Amtmann A, Armengaud P (2010) Potassium deficiency induces the biosynthesis of oxylipins and glucosinolates in Arabidopsis thaliana. BMC Plant Biology 10:172.

Vadassery J, Reichelt M, Hause B, Gershenzon J, Boland W, Mithöfer A (2012) CML42mediated calcium signaling coordinates responses to Spodoptera herbivory and abiotic stresses in Arabidopsis. Plant Physiology. 159:1159-1175

Wang M, Zheng Q, Shen Q, Guo S (2013) The critical role of potassium in plant stress response. International Journal of Molecular Sciences 14:7370-7390.

Wasternack C, Hause B (2013) Jasmonates: Biosynthesis, perception, signal transduction and action in plant stress response, growth and development. An update to the 2007 review in annals of botany. Annals of Botany 111:1021-1058.

Wasternack C, Song S (2017) Jasmonates: biosynthesis, metabolism, and signaling by proteins activating and repressing transcription. Journal of Experimental Botany 68:1303-1321.

Wu H, Ye H, Yao R, Zhang T, Xiong L (2015) OsJAZ9 acts as a transcriptional regulator in jasmonate signaling and modulates salt stress tolerance in rice. Plant Science 232:1-12.

Wu W, Peters J, Berkowitz GA (1991) Surface charge-mediated effects of $\mathrm{Mg}^{2+}$ on $\mathrm{K}^{+}$flux across the chloroplast envelope are associated with regulation of stromal $\mathrm{pH}$ and photosynthesis. Plant Physiology 97:580-587.

Yamada S, Kano A, Tamaoki D, Miyamoto A, Shishido H, Miyoshi S, Taniguchi S, Akimitsu $\mathrm{K}$, Gomi K (2012) Involvement of OsJAZ8 in jasmonate-induced resistance to bacterial blight in rice. Plant Cell Physiology 53:2060-72.

Yang DL, Yao J, Mei CS et al (2012) Plant hormone jasmonate prioritizes defense over growth by interfering with gibberellin signaling cascade. Proceedings of the National Academy of Sciences 109:E1192-1200.

Ye H, Du H, Tang N, Li X, Xiong L (2009) Identification and expression profiling analysis of TIFY family genes involved in stress and phytohormone responses in rice. Plant Molecular Biology 71:291-305. 
Figure 1. Promoter of $\boldsymbol{O s J A Z 9}$ is $\mathrm{K}$ deficiency responsive. GUS expression in lateral roots on nodal roots of $p O s J A Z 9: G U S$ transgenic rice plants. Expression was mainly appeared in laterals which was monitored at different time points under $\mathrm{K}$ deficiency. pOsJAZ9:GUS expression faded away as the $\mathrm{K}$ deficiency is prolonged from 3 days to 15 days. Uniformly germinated fifteen-days-old seedlings from $+K$ media were transferred to $-K$ media in a hydroponic system and GUS signal was analysed. (DAT, Day after K deficiency treatment).

Figure 2. Potassium deficiency causes a rapid increase in $\mathbf{J A}$ signaling in rice. (A) Schematic representation of OSJAZ9_GUS and OSJAZ9DC_GUS (Jas domain deleted) translational reporter constructs. (B) JA dependent proteasomal degradation of OsJAZ9 through Jas domain. Root tips of plants overexpressing OsJAZ9_GUS and OsJAZ9DC_GUS were analysed for GUS activity after $3 \mathrm{~h}$ of treatment with $100 \mu \mathrm{M}$ Me-JA alone or combined with $100 \mu \mathrm{M}$ proteasome inhibitor, MG132. (C) 15-days-old rice seedlings expressing OsJAZ9_GUS and OsJAZ9DC_GUS were transferred to K deficient media for indicated time points $(0.5$ to $12 \mathrm{~h})$. Stability of OsJAZ9_GUS and OsJAZ9AC_GUS proteins in response to $\mathrm{K}$ deficiency were analyzed by histochemical GUS staining of roots. The experiment was performed in three independent replicates.

Figure 3. Enhanced K acquisition in K-starved OsJAZ9 OE seedlings upon K resupply. (A) Morphological data (root dry weight, shoot dry weight and number of roots per plant) of 10days-old normally grown seedlings (normal/S1), (B) followed by 15 -days of $\mathrm{K}$ starvation (K stress/S2) and (C) subsequent recovery with $10 \mu \mathrm{M} \mathrm{K}_{2} \mathrm{SO}_{4}$ for 5 days (Recovery/S3). K stress represents the ratio of dry root, or shoot biomass $\mathrm{K}$ stressed plants with respect to normal condition and recovery represents the ratio of recovered plants with respect to normal condition $(\mathrm{n}=<15)$. (D) $\mathrm{Na}^{+} / \mathrm{K}^{+}$and (E) Total $\mathrm{K}$ content per plant of WT and OsJAZ9 transgenics during $\mathrm{S} 1, \mathrm{~S} 2$ and S3 treatments. K content experiment was performed with two replicates. Asterisks indicate significant changes in transgenics compared to WT at respective time points $(p \leq 0.05$, .01 and .001 for $*, * *$ and $* * *$ calculated from Student's $t$-test). Scale $=10 \mathrm{~cm}$.

Figure 4. JA-induced root inhibition in WT and $O S J A Z 9$ transgenics. (A) Representative images of roots of 20-days-old WT, RN12 and OE10 seedlings supplemented with DMSO (Mock; M) or $10 \mu \mathrm{M}$ MeJA dissolved in DMSO (JA treated; J). Scale $=5 \mathrm{~cm}$. (B) Average root 
lengths of WT, OsJAZ9 RNAi and OE plants under DMSO (blue bars) and MeJA (Red bars) treatments. Each bar represents average of 9 seedlings with standard error. Values over the bars represent JA mediated percentage inhibition in the root length as compared to DMSO treated plants. Significant differences between DMSO vs MeJA treatment were evaluated by Student's $t$-test. Asterisks; *, **, *** and **** indicate $p$ values, $\leq 10-3,10-4,10-8$ and 10-10, respectively.

Figure 5. Overexpression of $O S J A Z 9$ enhances $\mathrm{K}$ deficiency response in rice. (A) Venn diagrams showing differentially regulated genes on $\mathrm{K}$ deficiency in WT and OsJAZ9 OE lines after 15 days of deficiency. (B) Overview of transcriptome data showing category wise distribution of differentially genes in WT and OsJAZ9 OE lines during $\mathrm{K}$ deficiency. Error bar represents SE of all the replicates. ( $p \leq 0.05$, Student's $t$-test). FC=Fold Change.

Figure 6. OsJAZ9 overexpression influences root system architecture. (A) Representative root images of 12-days-old WT and OsJAZ9 transgenics showing lateral root lengths during K deficient (upper panel) and K sufficient conditions (lower panel). (B) Average seminal root length and (C) Average lateral root length of WT and OsJAZ9 transgenics during K deficient (upper row) and K sufficient (lower row) conditions. Four independent plants were used for the analysis. Scale bar represents $1 \mathrm{~cm}$ while error bar represents SE among the replicates. ( $p$ $\leq 0.05$, Student's $t$-test).

Figure 7. OsJAZ9 OE lines accumulate higher JA-Ile than WT under K deficiency. JA-Ile content in WT and OsJAZ9 OE (OE10) lines during normal $(+\mathrm{K})$ and 15 days of K deficiency $(-\mathrm{K})$ conditions. Bars represent the average change in JA-Ile content among at least four replicates. Error bar represents SE of all the replicates. ( $p \leq 0.05$, Student's $t$-test). FC=Fold Change.

\section{Supporting Information}

Figure S1. Effect of K deficiency on rice growth.

Figure S2. Potassium deficiency induces JA signaling in rice.

Figure S3. Nuclear localization of YFP-OsJAZ9.

Figure S4. Effect of prolonged K deficiency on OsJAZ9 stability. 
Figure S5. Raising and screening of OSJAZ9 expressing rice transgenics.

Figure S6. Effect of OsJAZ9 overexpression on growth parameters of rice during K sufficient and deficient conditions.

Figure S7. Effect of OsJAZ9 knockdown on growth parameters of rice during K sufficient and deficient conditions.

Figure S8. Overexpression of OSJAZ9 enhances expression of $\mathrm{K}^{+}$transporters.

Figure S9. Validation of RNA-seq data with qRT-PCR.

Figure S10. Tissue-specific expression of OsJAZ9.

Figure S11. Exogenous application of MeJA modulates root system architecture under K sufficient conditions.

Figure S12. Exogenous application of MeJA modulates root system architecture under K deficient conditions.

Figure S13. Raising and screening of OsJAZ9 OE rice transgenic lines with ZmUbi1 promoter.

Figure S14. OsJAZ9 is involved in $\mathrm{K}$ deficiency tolerance in rice.

Figure S15. OsJAZ9 overexpression influences root system architecture.

Figure S16. JA content in WT and OsJAZ9 OE (OE10) plants during normal (+K) and 15 days of $\mathrm{K}$ deficiency $(-\mathrm{K})$ conditions.

Supporting Table S1. List of primers used for qRT-PCR and gene cloning.

Supporting Table S2. Effect of K deficiency on expression of JA associated genes.

Supporting Table S3. Effect of OSJAZ9 expression on expression of JA associated genes under K deficiency.

Supporting Table S4. Effect of OsJAZ9 expression on K transporter expression under K deficiency.

Supporting Table S5. Effect of OsJAZ9 expression on expression of $O S J A Z$ genes. 


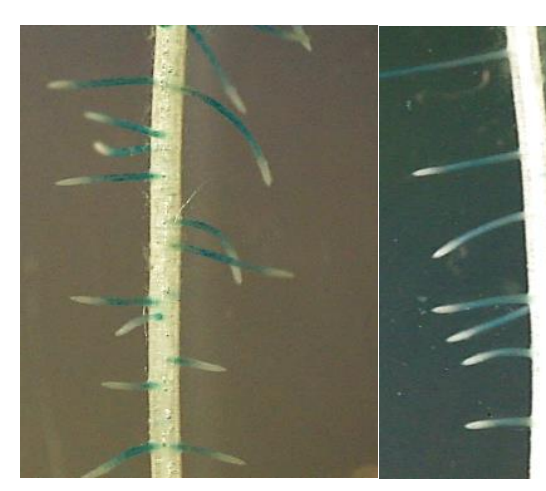

$+\mathrm{K}$

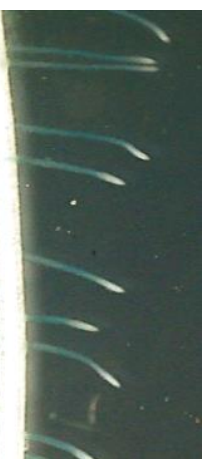

-K 3 DAT

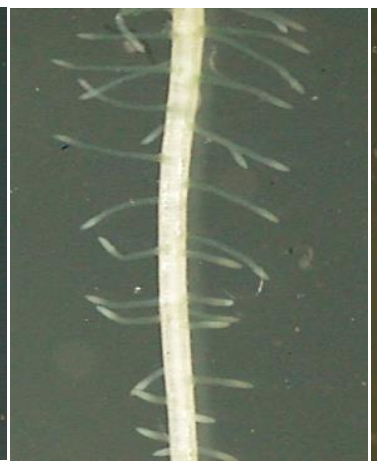

-K 5 DAT

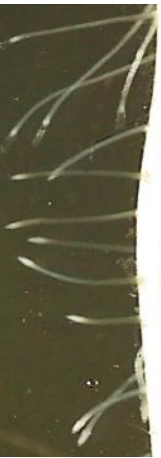

-K 10 DAT
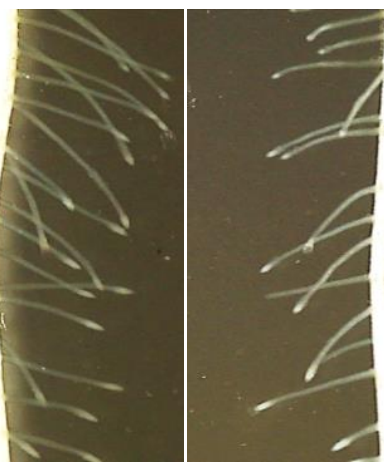

-K 15 DAT

Figure 1. Promoter of $\boldsymbol{O S J A Z 9}$ is $\mathbf{K}$ deficiency responsive. Gus expression in lateral roots on nodal roots of $p O s J A Z 9: G U S$ transgenic rice plants. Expression was mainly appeared in laterals which was monitored at different time points under $\mathrm{K}$ deficiency. pOsJAZ9:GUS expression faded away as the $\mathrm{K}$ deficiency is prolonged from 3 days to 15 days. Uniformly germinated fifteen-days-old seedlings from $+\mathrm{K}$ media were transferred to $-\mathrm{K}$ media in a hydroponic system and gus signal was analysed. (DAT, Day after K deficiency treatment). 
(A)

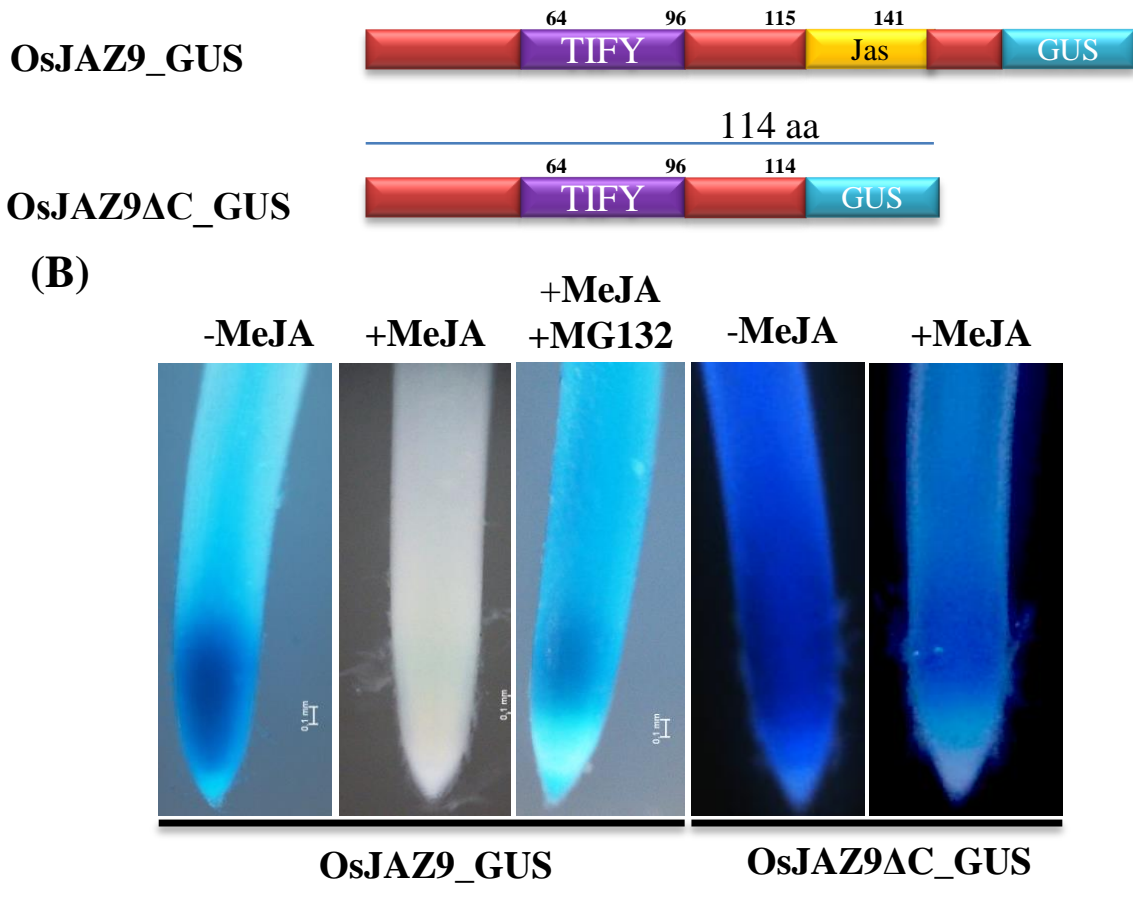

(C)

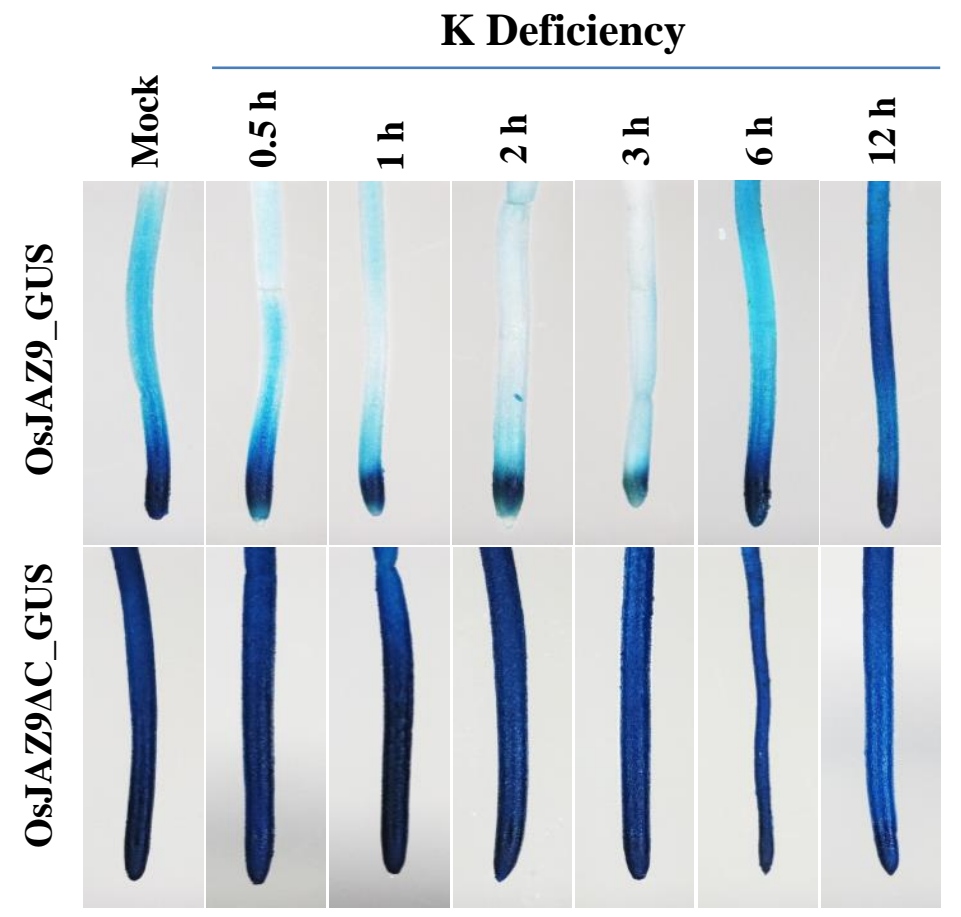

Figure 2. Potassium deficiency causes a rapid increase in JA signaling in rice. (A) Schematic representation of OsJAZ9_GUS and OsJAZ9DC_GUS (Jas domain deleted) translational reporter constructs. (B) JA dependent proteasomal degradation of OsJAZ9 through Jas domain. Root tips of plants overexpressing OsJAZ9_GUS and OSJAZ9AC_GUS were analysed for GUS activity after $3 \mathrm{~h}$ of treatment with $100 \mu \mathrm{M} \mathrm{Me}-$ $\mathrm{JA}$ alone or combined with $100 \mu \mathrm{M}$ proteasome inhibitor, MG132. (C) 15-days-old rice seedlings expressing OsJAZ9_GUS and OsJAZ9AC_GUS were transferred to K deficient media for indicated time points ( 0.5 to 12 h). Stability of OsJAZ9_GUS and OsJAZ9AC_GUS proteins in response to K deficiency were analyzed by histochemical GUS staining of roots. The experiment was performed in three independent replicates. 
(A)

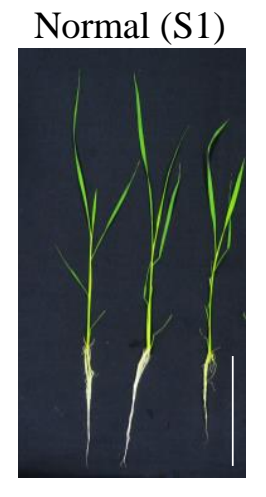

$\mathrm{K}$ stress (S2)

(B)

(C)
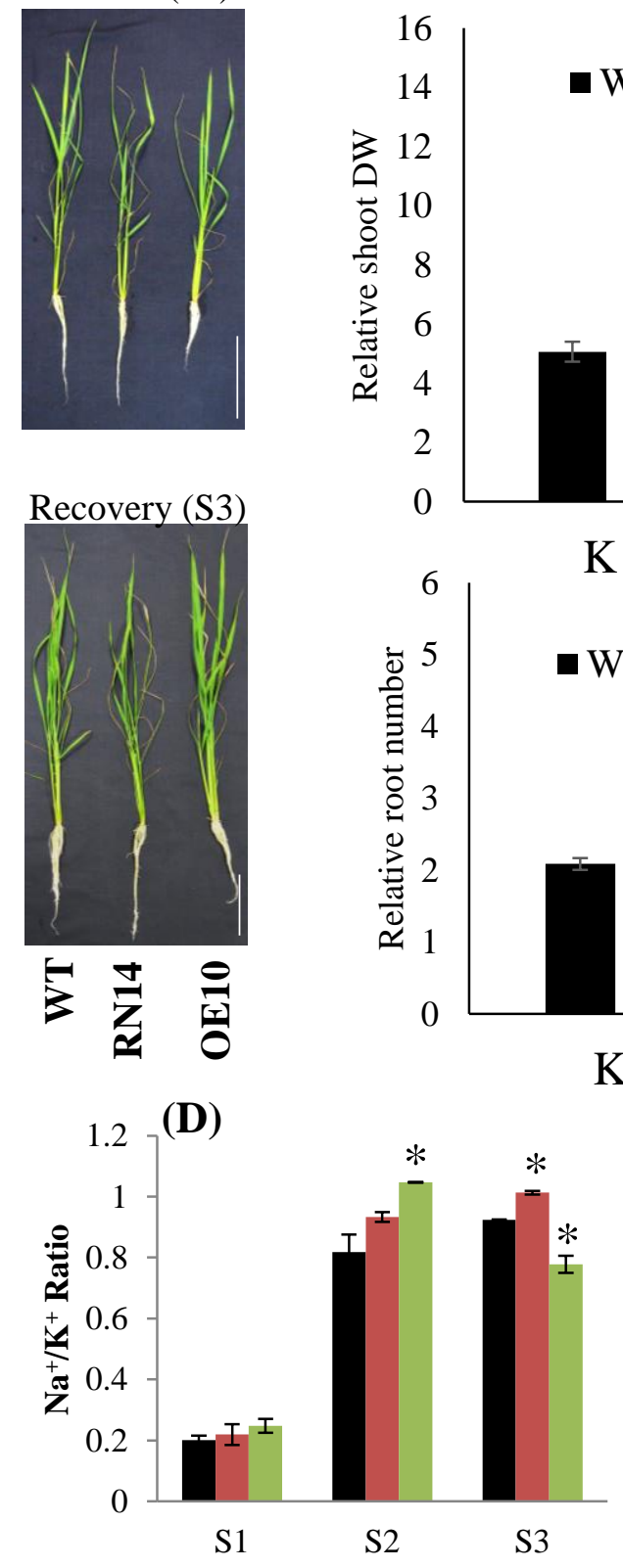
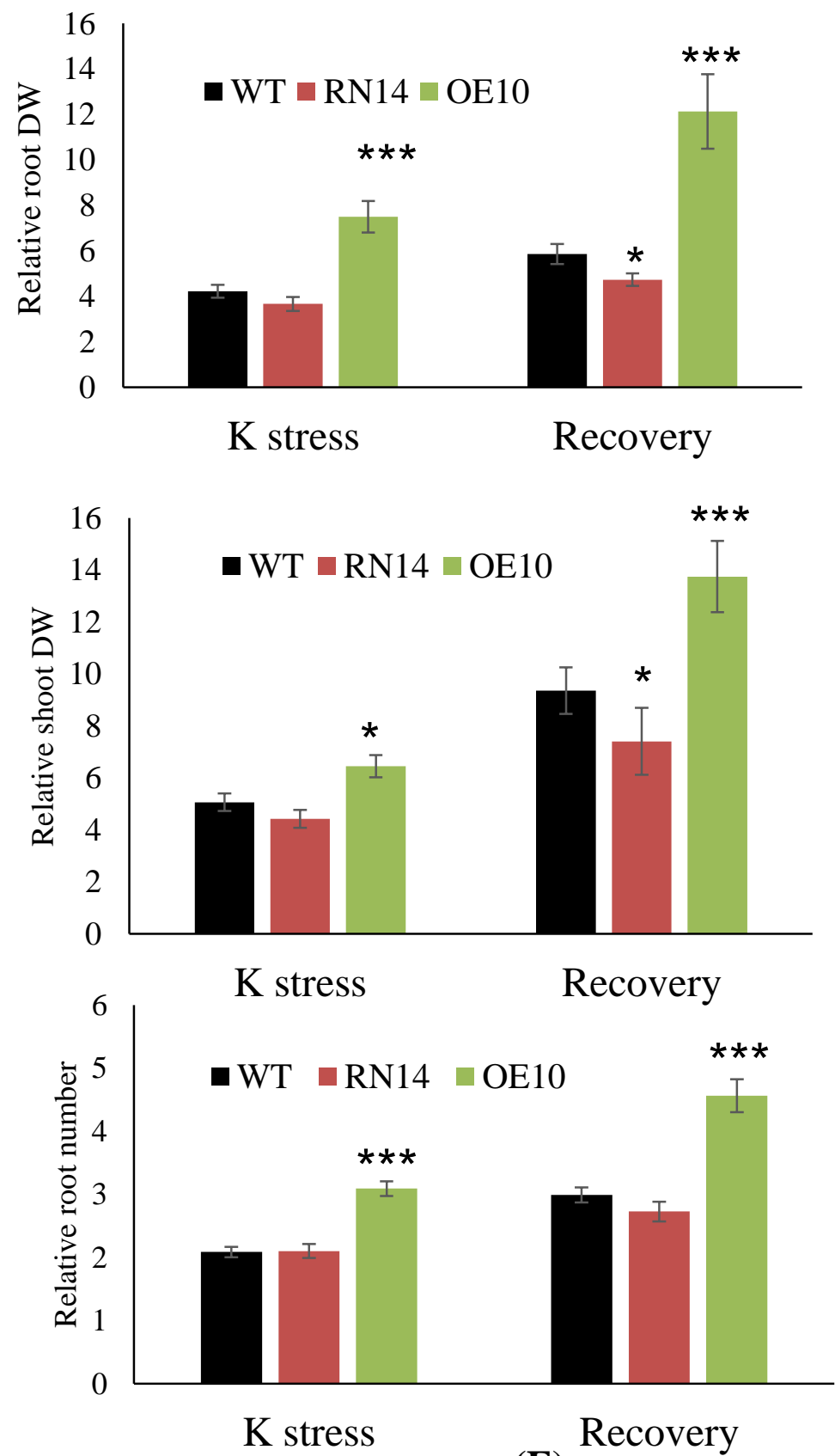

(E)

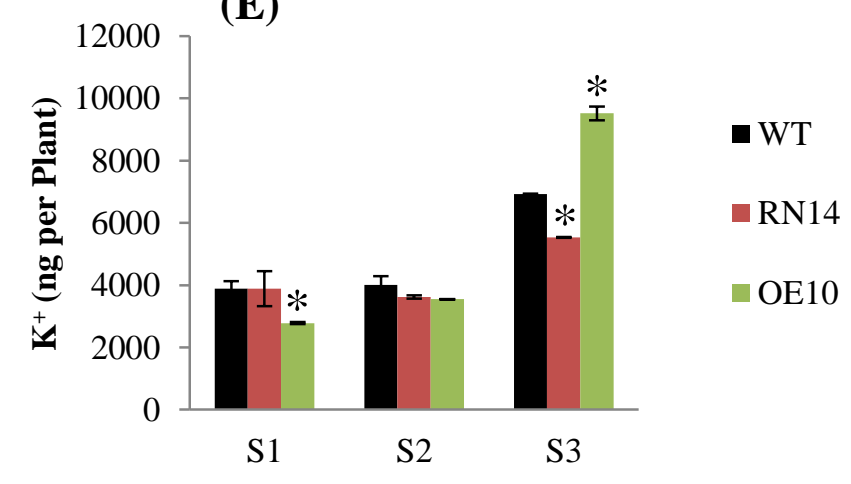

Figure 3. Enhanced K acquisition in K-starved OSJAZ9 OE seedlings upon K resupply. (A) Morphological data (root dry weight, shoot dry weight and number of roots per plant) of 10-days-old normally grown seedlings (normal/S1), (B) followed by 15-days of $\mathrm{K}$ starvation ( $\mathrm{K}$ stress/S2) and (C) subsequent recovery with $10 \mu \mathrm{M} \mathrm{K}_{2} \mathrm{SO}_{4}$ for 5 days (Recovery/S3). K stress represent the ratio of dry root or shoot biomass $\mathrm{K}$ stressed plants with respect to normal condition and recovery represent the ratio of recovered plants with respect to normal condition $(\mathrm{n}=<15)$. (D) $\mathrm{Na}^{+} / \mathrm{K}^{+}$and (E) Total $\mathrm{K}$ content per plant of WT and OsJAZ9 transgenics during S1, S2 and S3 treatments. K content experiment was performed with two replicates. Asterisks indicate significant changes in transgenics compared to WT at respective time points $(p \leq$ $0.05, .01$ and .001 for $* * *$ and $* * *$ calculated from Student's $t$-test). Scale $=10 \mathrm{~cm}$. 
(A)

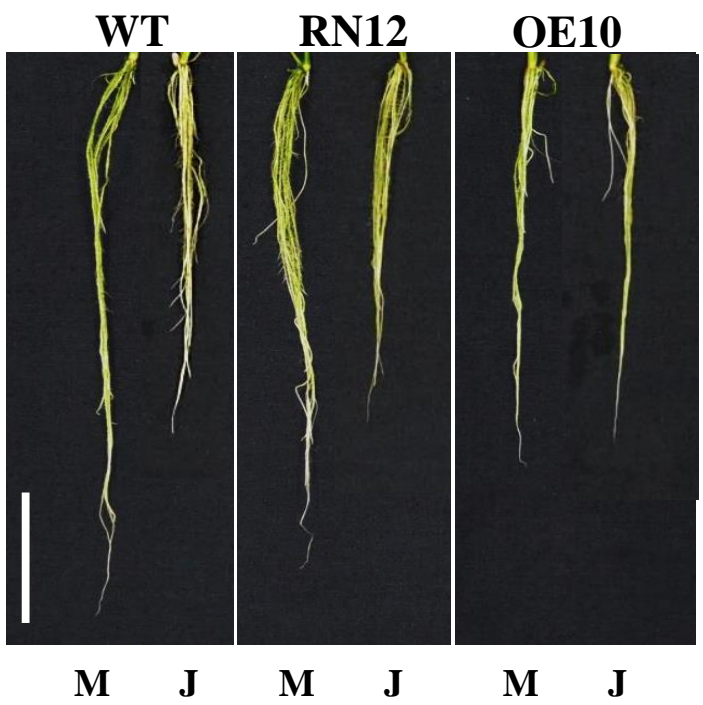

(B)

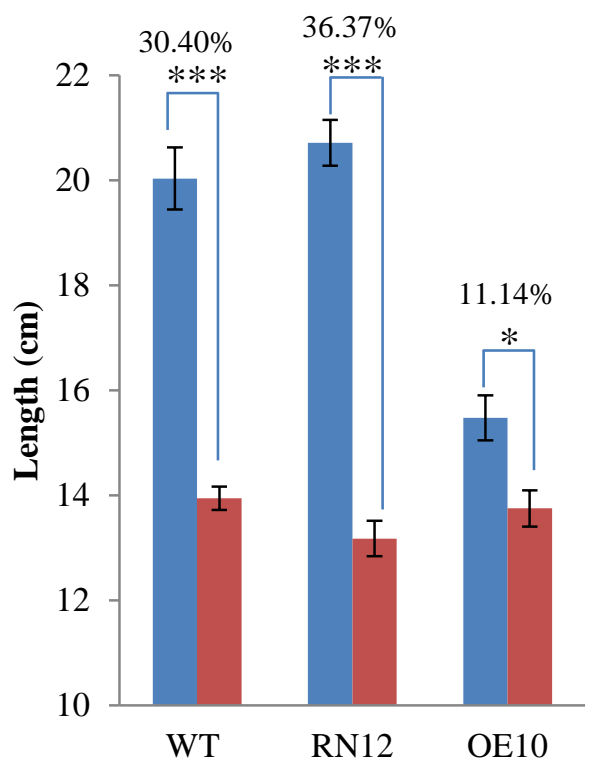

Figure 4. JA-induced root inhibition in WT and $O S J A Z 9$ transgenics. (A) Representative images of roots of 20-days-old WT, RN12 and OE10 seedlings supplemented with DMSO (Mock; M) or $10 \mu \mathrm{M}$ MeJA dissolved in DMSO (JA treated; J). Scale $=5 \mathrm{~cm}$. (B) Average root lengths of WT, OsJAZ9 RNAi and OE plants under DMSO (blue bars) and MeJA (Red bars) treatments. Each bar represents average of 9 seedlings with standard error. Values over the bars represent JA mediated percentage inhibition in the root length as compared to DMSO treated plants. Significant differences between DMSO vs MeJA treatment were evaluated by Student's $t$-test. Asterisks; $*, * *, * * *$ and $* * * *$ indicate $p$ values, $\leq 10-3,10-4,10-8$ and $10-10$, respectively. 
(A)

WT+K Vs WT-K OE+K Vs OE-K

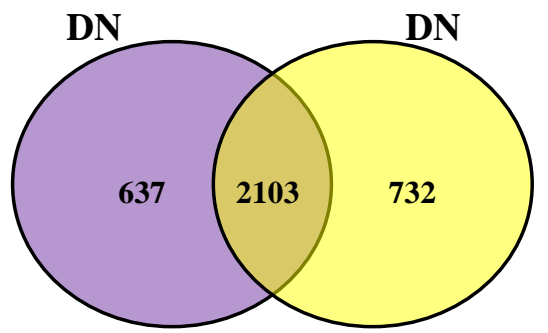

WT+K Vs WT-K OE+K Vs OE-K

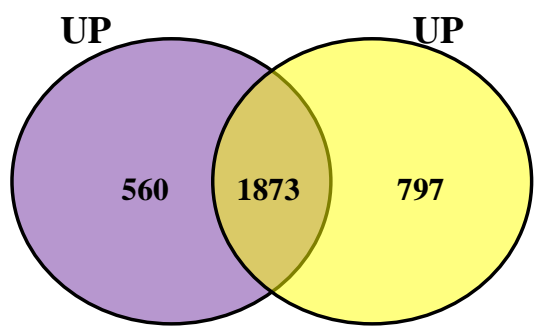

(B)

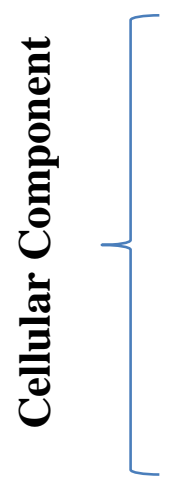

光

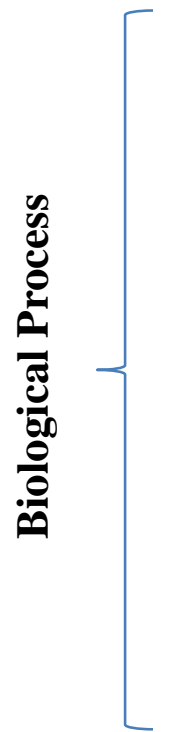

चT+K Vs WT-K

- OE+K Vs OE -K endoplasmic reticulum
cytoplasm cell wall

cell

transporter translation transferase activity structural molecule activity signal transduction signal transducer activity transcription factor epigenetic regulation protein binding nucleotide binding nucleic acid binding nuclease activity motor activity molecular function lipid binding kinase activity hydrolase activity enzyme regulator activity DNA binding catalytic activity binding

transport secondary metabolic process response to stress response to biotic stimulus response to abiotic stimulus reproduction post-embryonic development pollen-pistil interaction photosynthesis metabolic process lipid metabolic process growth

flower development embryo development cellular process cellular homeostasis cell growth

cell differentiation cell death cell cycle catabolic process carbohydrate metabolic process biosynthetic process biological process

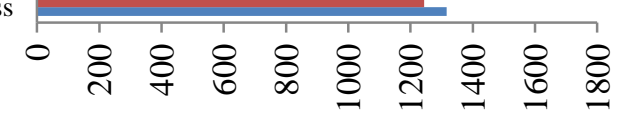

Number of Genes

Figure 5. Overexpression of OsJAZ9 enhances $\mathrm{K}$ deficiency response in rice. (A) Venn diagrams showing differentially regulated genes on K deficiency in WT and OsJAZ9 OE lines after 15 days of deficiency. (B) Overview of transcriptome data showing category wise distribution of differentially genes in WT and OsJAZ9 OE lines during K deficiency. Error bar represents SE of all the replicates. ( $p \leq 0.05$, Student's $t$-test). FC=Fold Change. 
(A)
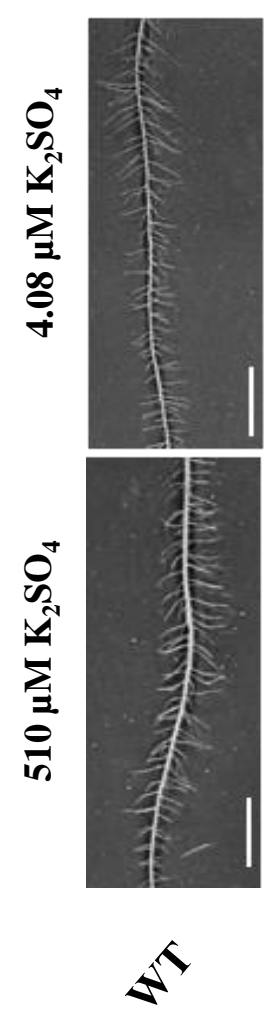

(B)
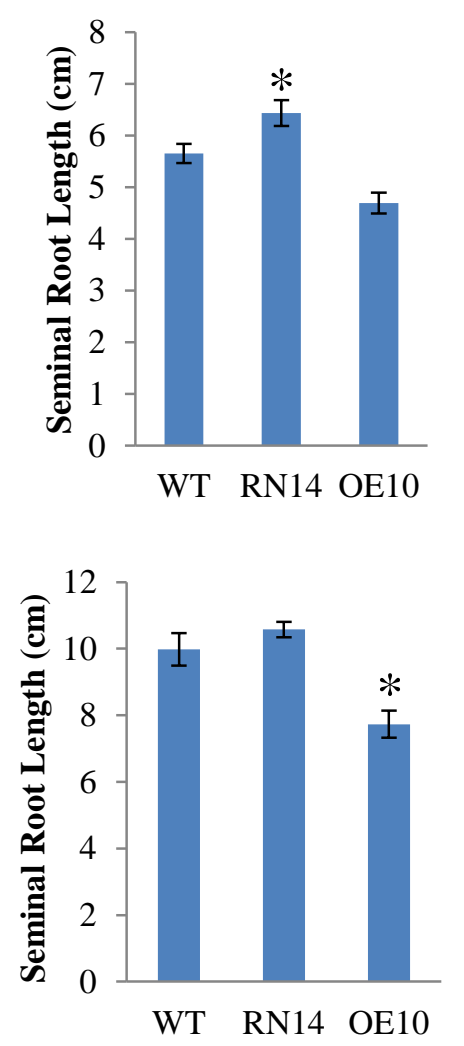

(C)
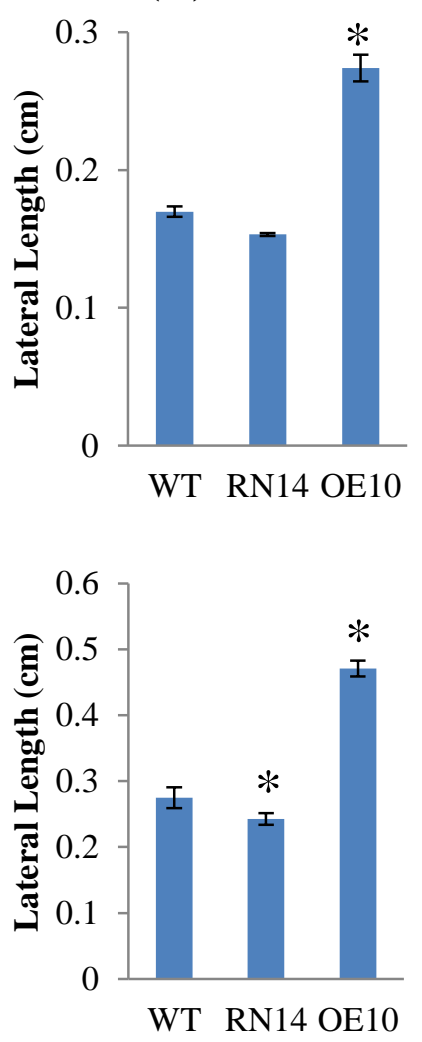

Figure 6. OSJAZ9 overexpression influences root system architecture. (A) Representative root images of 12-days-old WT and OsJAZ9 transgenics showing lateral root lengths during K deficient (upper panel) and $\mathrm{K}$ sufficient conditions (lower panel). (B) Average seminal root length and (C) Average lateral root length of WT and OsJAZ9 transgenics during K deficient (upper row) and $\mathrm{K}$ sufficient (lower row) conditions. Four independent plants were used for the analysis. Scale bar represents $1 \mathrm{~cm}$ while error bar represents SE among the replicates. ( $p \leq 0.05$, Student's $t$-test). 


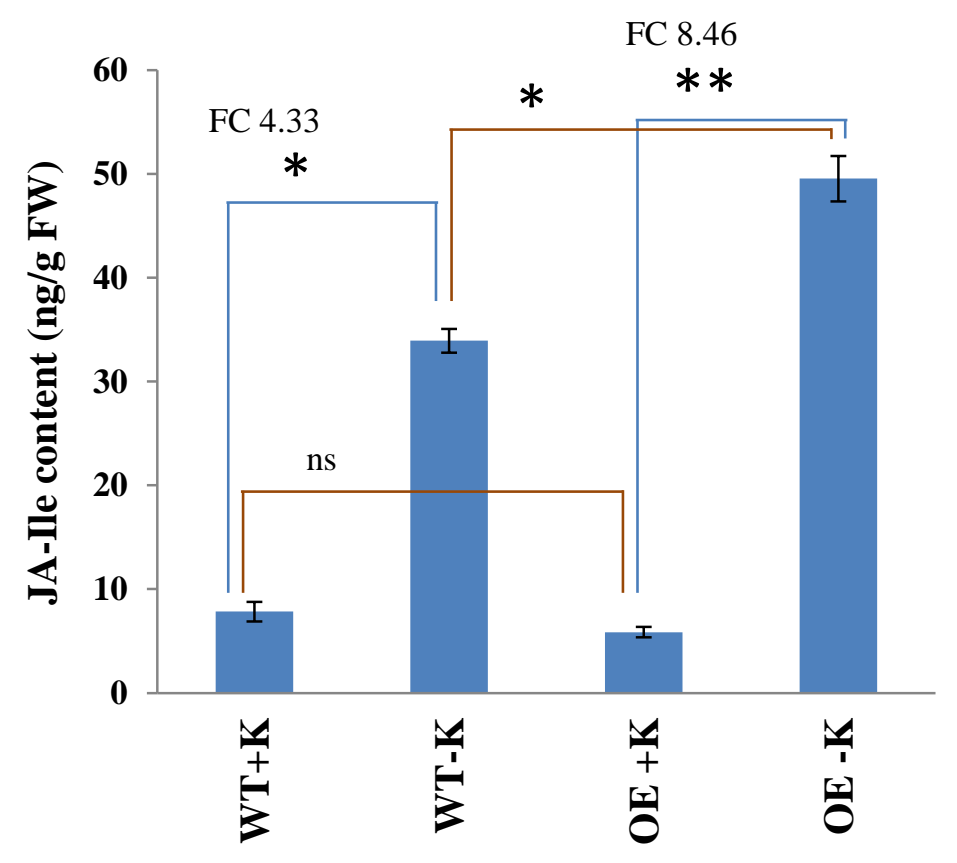

Figure 7. OsJAZ9 OE lines accumulate higher JA-Ile than WT under K deficiency. JA-Ile content in WT and OsJAZ9 OE (OE10) lines during normal $(+\mathrm{K})$ and 15 days of $\mathrm{K}$ deficiency (-K) conditions. Bars represent the average change in JA-Ile content among at least four replicates. Error bar represents SE of all the replicates. ( $p \leq 0.05$, Student's $t$-test). FC $=$ Fold Change. 


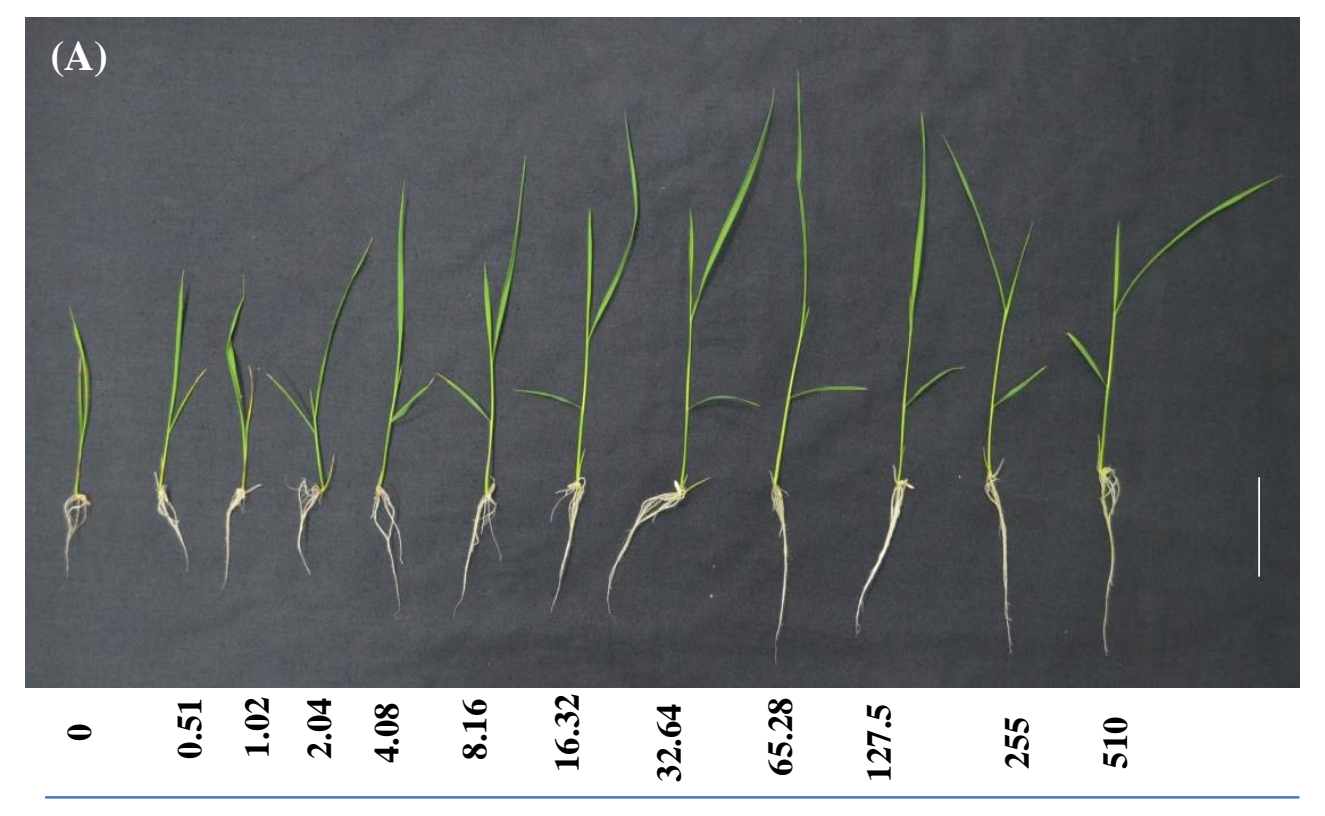

Conc. $\mathrm{K}_{2} \mathrm{SO}_{4}(\mu \mathrm{M})$

(B)

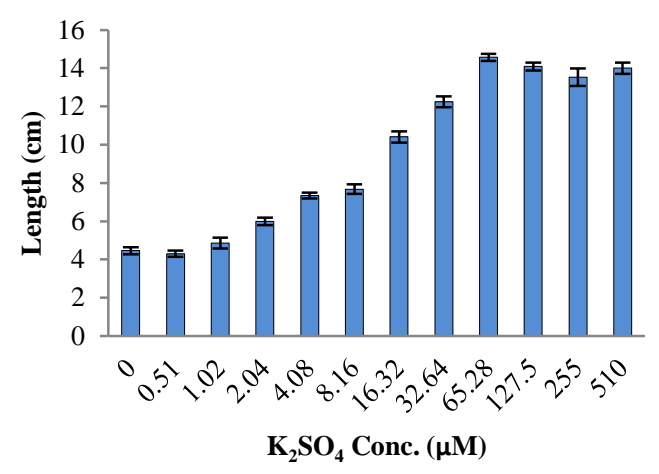

(D)

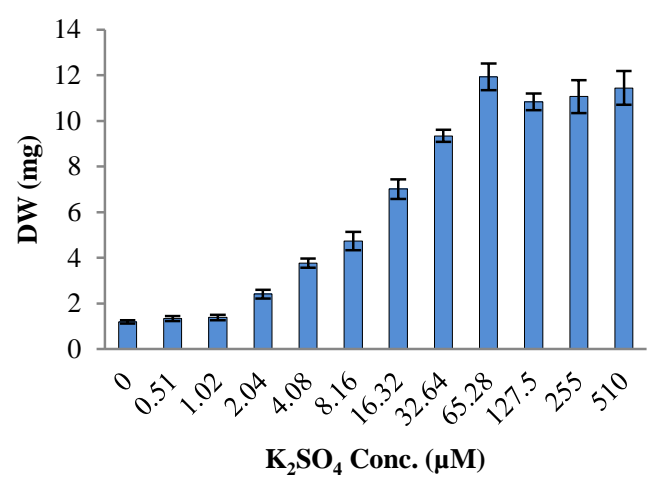

(C)

Shoot Length

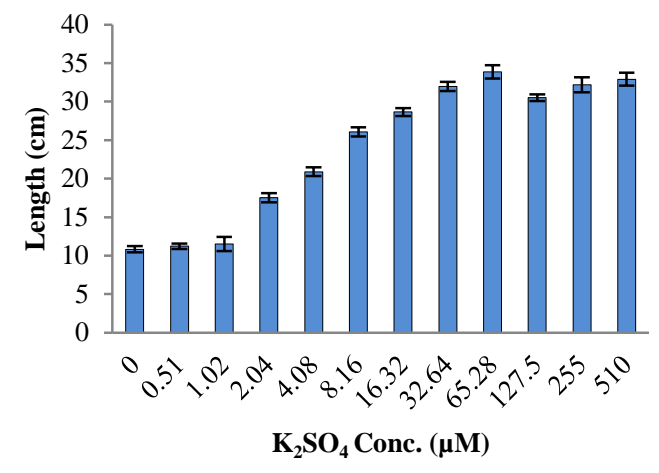

(E)

$$
\text { Shoot DW }
$$

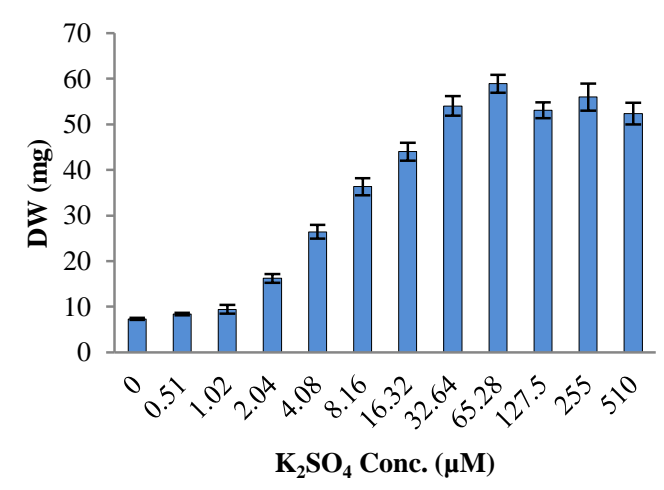

Figure S1. Effect of $\mathbf{K}$ deficiency on rice growth. (A) Representative images showing effect of different concentrations of $\mathrm{K}$ on plant growth. Uniformly germinated seedlings were transferred to Yoshida media containing different concentrations of $\mathrm{K}_{2} \mathrm{SO}_{4}$ (as indicated on $\mathrm{X}$-axis). (B) Root length, (C) Shoot length, (D) Root dry biomass and (E) Shoot dry biomass of rice seedlings after 15 days of growth under varied $\mathrm{K}$ supply. Each bar represents average of 10 seedlings with standard error. Scale = $5 \mathrm{~cm}$. 
(A)

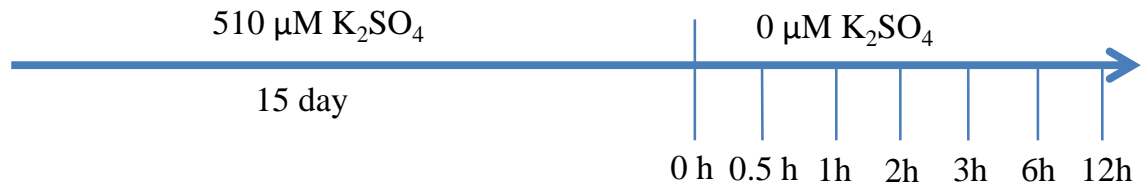

(B)
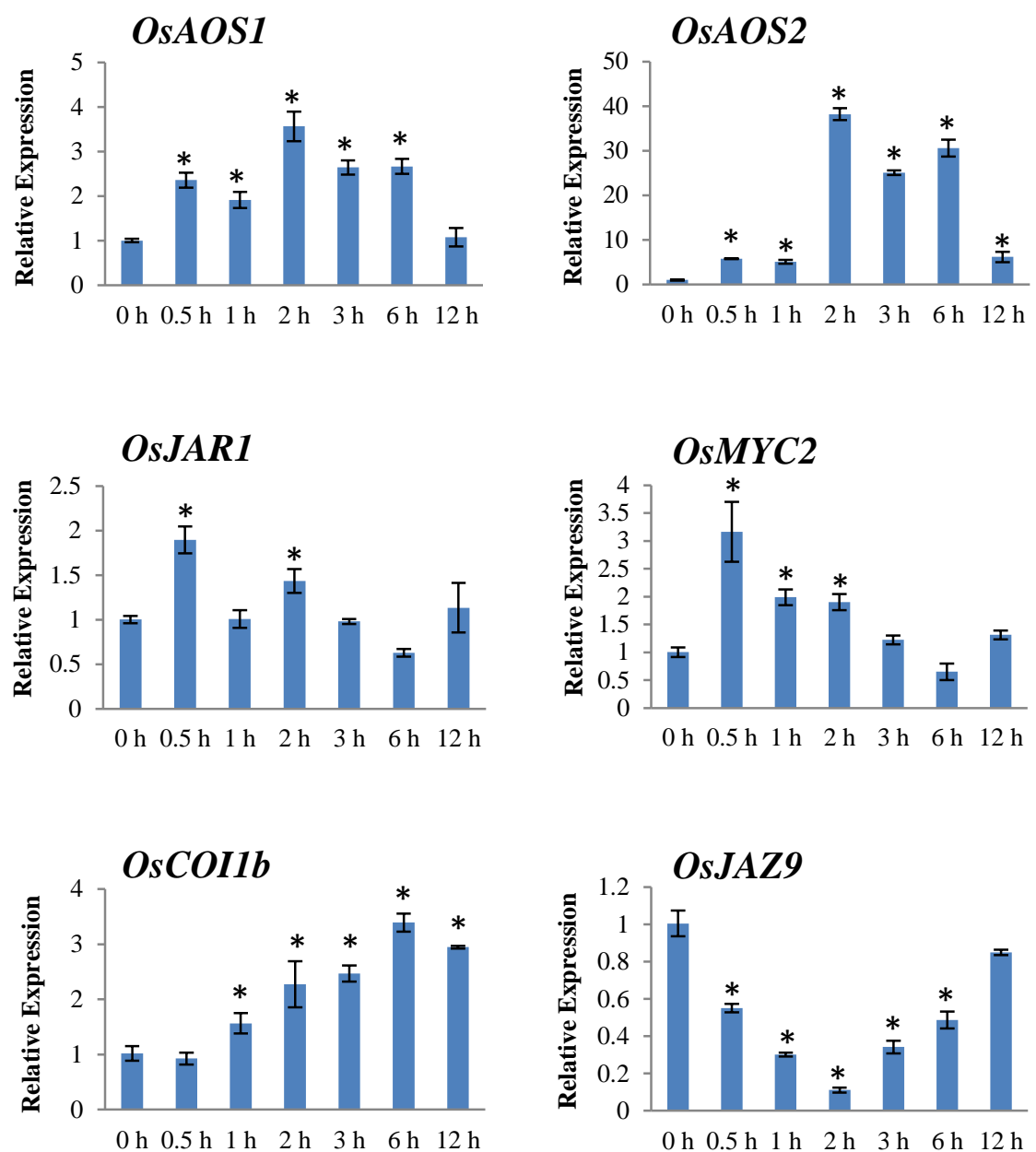

Figure S2. Potassium deficiency induces JA signaling in rice. (A) Schematic representation of experimental setup used for expression analysis of JA biosynthetic and signaling genes. Plants were raised under normal conditions $\left(510 \mu \mathrm{M} \mathrm{K} \mathrm{SO}_{4}\right)$ for 15 days before being subjected to different durations $(0.5$ to $12 \mathrm{~h})$ of $\mathrm{K}$ deficiency treatment $\left(0 \mu \mathrm{M} \mathrm{K}_{2} \mathrm{SO}_{4}\right)$. (B) Short-term $\mathrm{K}$ deficiency responses of JA biosynthetic and signaling genes. Relative expression levels at different time points of $\mathrm{K}$ deficiency were analysed with respect to $0 \mathrm{~h}$. Error bar indicates standard error derived from three independent replicates. Asterisk indicates significant changes at different time points compared to $0 \mathrm{~h}$ treatment $(p \leq 0.05$, Student's $t$-test). 
(A)

Plant-mPLoc:Predicting subcellular localization of plant proteins including those with multiple sites

| Read Me $\mid$ Data | Citation

Your input sequence (179aa) is:

$>$ OSJAZ9

MASTDPMTRRFAVACGVLSQYVKANSSQPSTAAPVAQGVSGLMAAAAAAAAAPVVQEPGC

EVDGGGQQFTIFYAGKVVVIDRCTPAMAAELMRFASAAQGGGGAPEAPPAL VDMPIARKA

Plant-mPLoc Computation Result

Query protein Predicted location(s)

OsJAZ9 Nucleus.

(B)

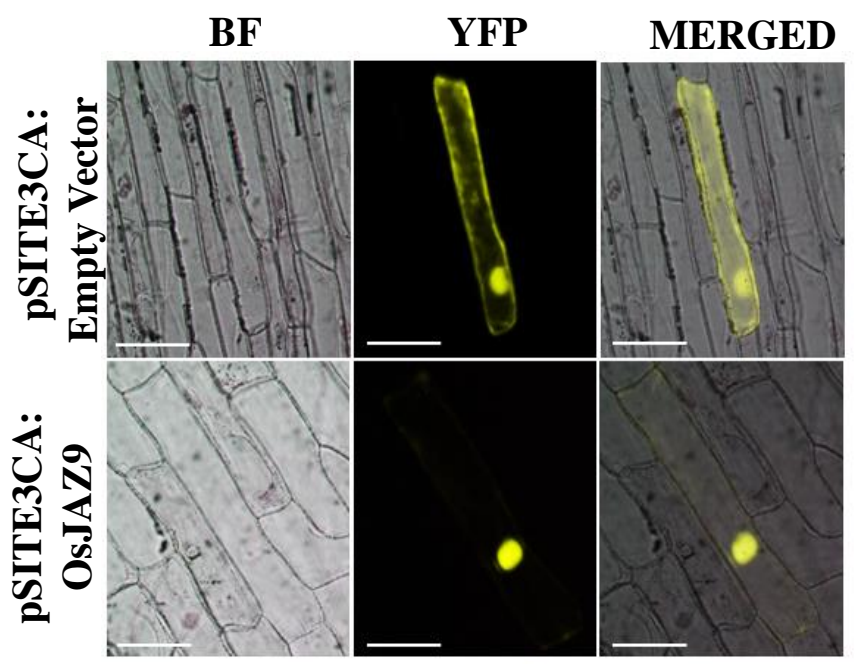

Figure S3. Nuclear localization of YFP-OsJAZ9. (A) In-silico prediction of OsJAZ9 localization using databases

(http://www.csbio.sjtu.edu.cn/cgi-bin/PlantmPLoc.cgi).

(B) Subcellular localization of YFP-OsJAZ9 in onion epidermal cells. Merged images showing superimposition of YFP and Bright field (BF) images. 
(A)

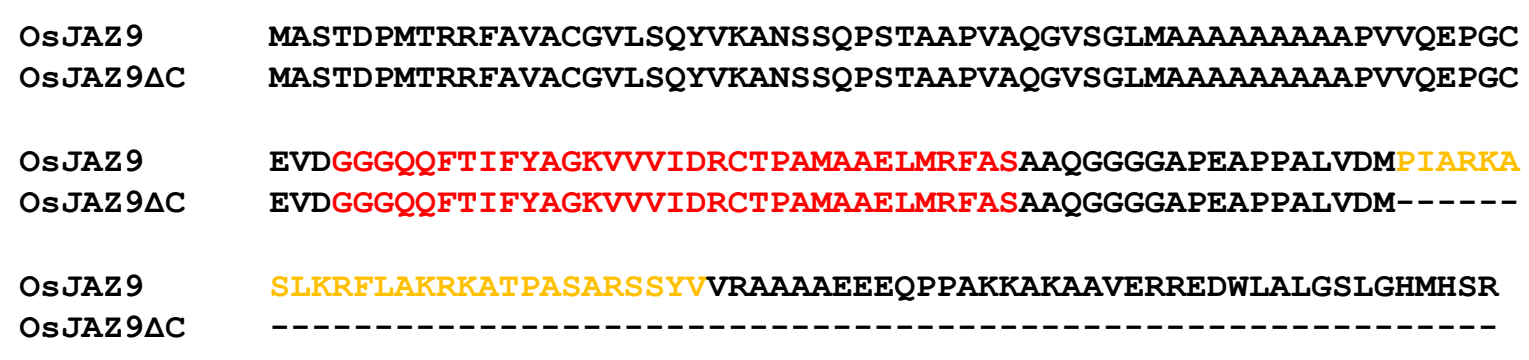

(B)
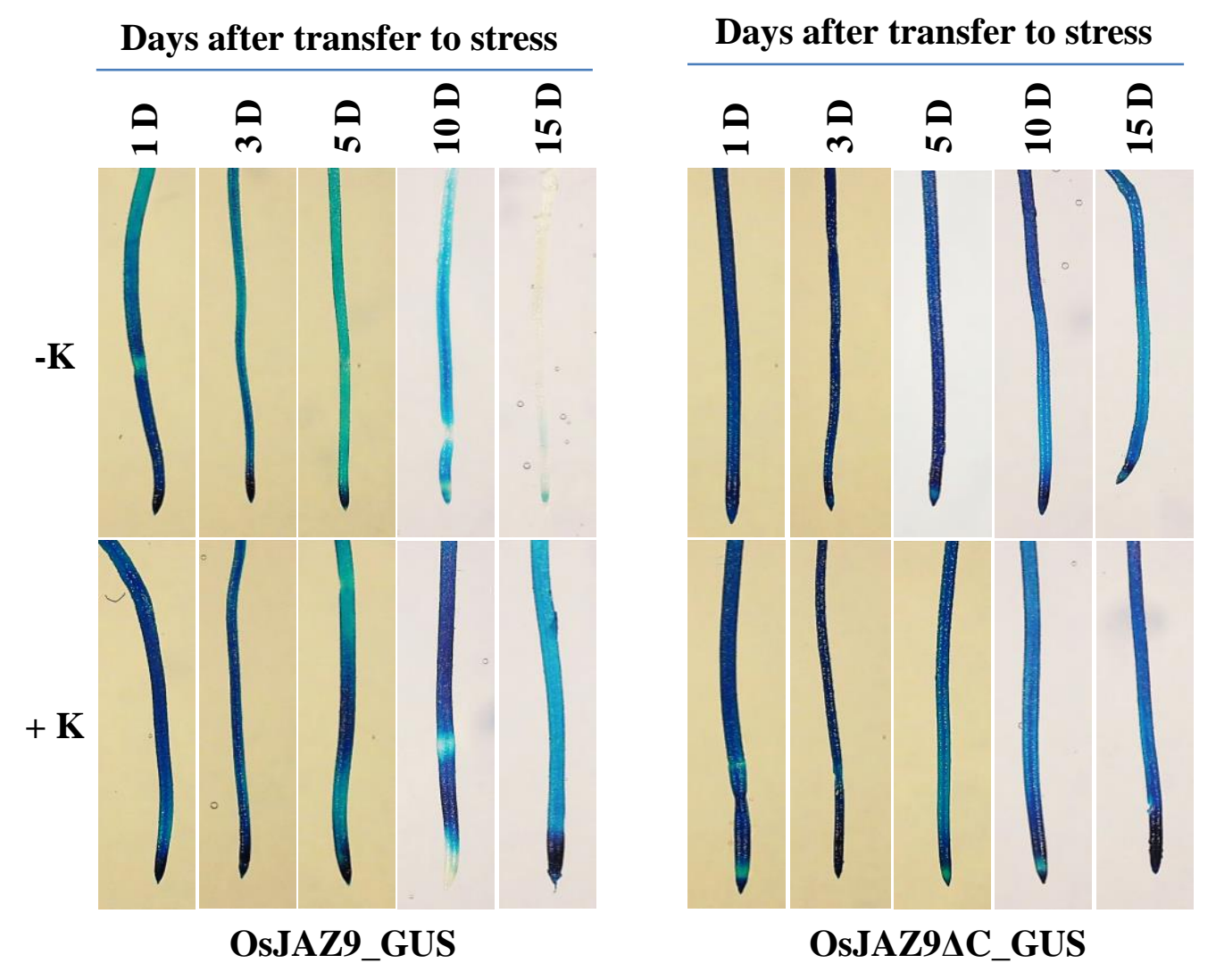

Figure S4. Effect of prolonged $K$ deficiency on OsJAZ9 stability. (A) Protein sequence alignment of OsJAZ9 and OsJAZ9 $\triangle$ C. Red text highlights TIFY domain while the yellow text highlights Jas motif. (B) OsJAZ9_GUS degradation on prolonged $\mathrm{K}$ deficiency. 15-days-old rice seedlings expressing OSJAZ9_GUS and OSJAZ9DC_GUS were transferred to K deficient media for indicated time points ( 1 day to 15 days). Stability of OsJAZ9_GUS and OsJAZ9AC_GUS proteins in response to $\mathrm{K}$ deficiency was analysed by histochemical GUS staining of root sections. Experiment was performed in three independent replicates. 


\section{(A)}

Vector constructs for $O S J A Z 9$ overexpression p35S:OsJAZ9_GUS

LB CaMV poly(A)
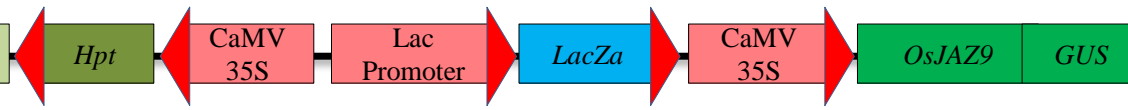
\begin{tabular}{|l|}
\hline $6 \mathrm{X}$ \\
$\mathrm{His}$ \\
\hline
\end{tabular}

p35S:OsJAZ9AC_GUS

LB CaMV poly(A)
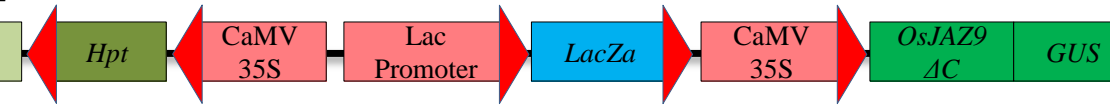

Vector constructs for OsJAZ9 silencing

LB

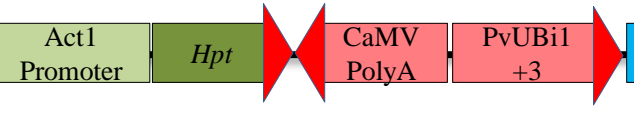

Vector constructs for pOSJAZ9:GUS reporter

$\mathrm{RB}$
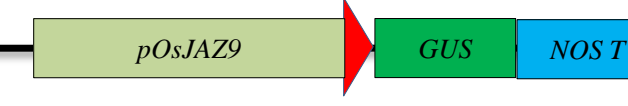

\section{LB}

(B)
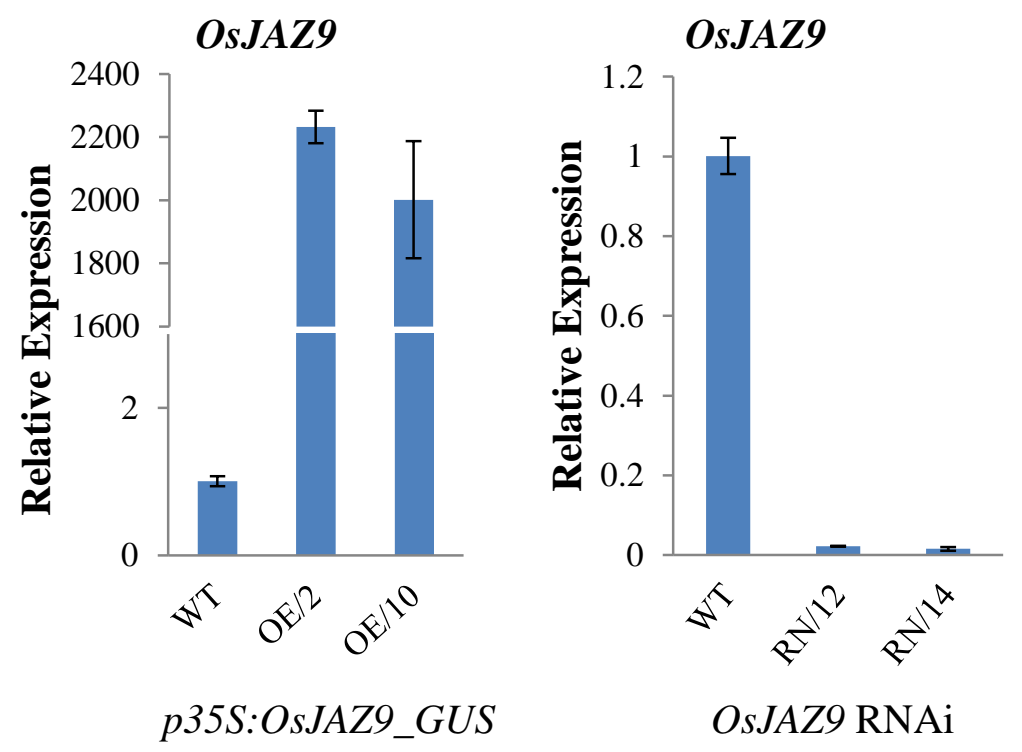

Figure S5. Raising and screening of $O \boldsymbol{S} J A Z 9$ expressing rice transgenics. (A) Schematic of OsJAZ9 overexpression and silencing constructs. (B) Relative expression of OSJAZ9 in overexpression (p35S:OsJAZ9_GUS) and RNAi lines. Gene expression analysis was done with qRT-PCR with at least three independent replicates. Asterisk indicates significant change compared to WT ( $p \leq 0.05$, Student's $t$ test). 

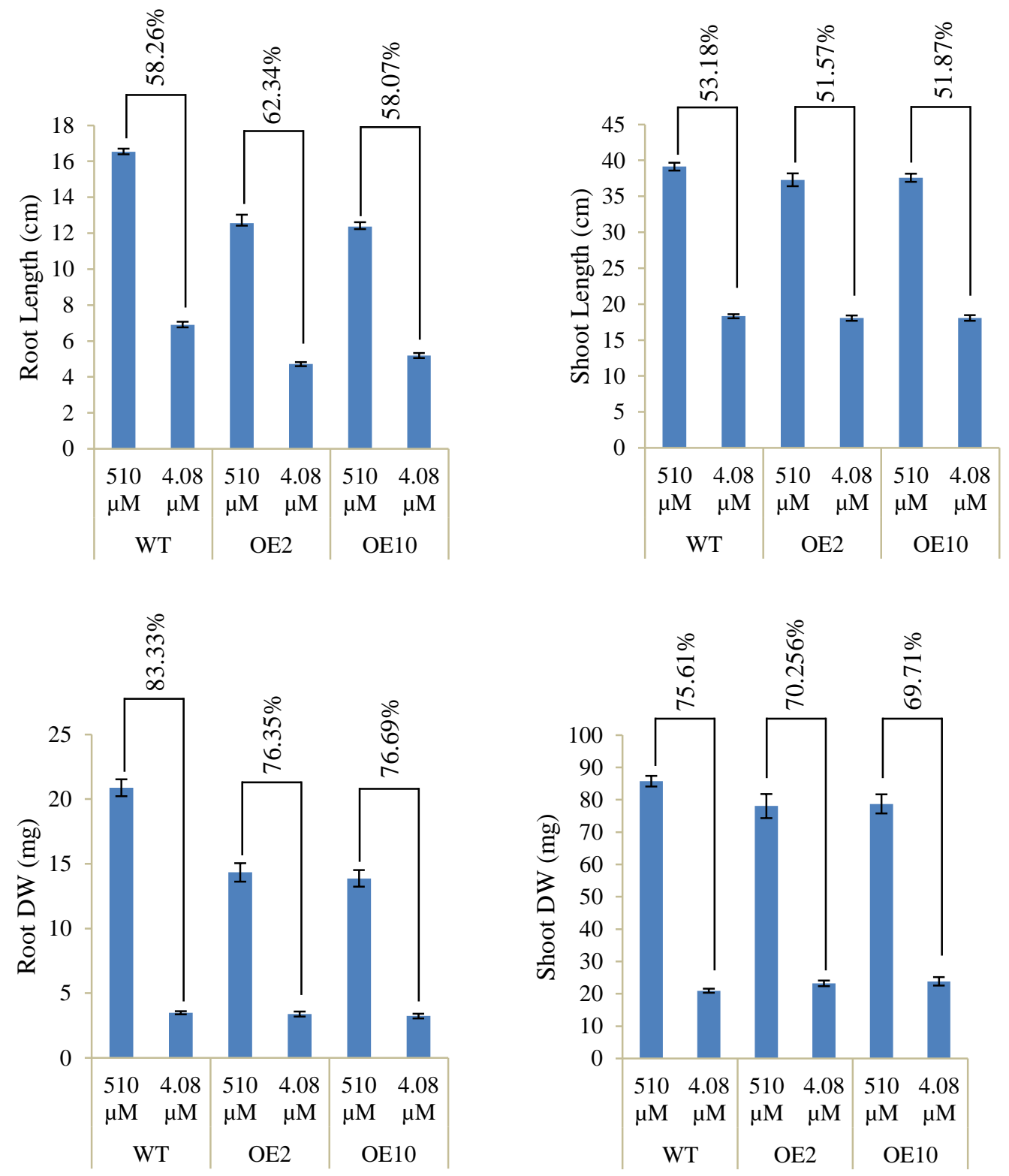

Figure S6. Effect of $O S J A Z 9$ overexpression on growth parameters of rice during K sufficient and deficient conditions. Growth parameters (length and dry biomass of root and shoot) of WT and OsJAZ9 OE (OE2 and OE10) lines under K deficient and sufficient conditions. Each bar represents average of 12 seedlings with standard error while the values above the bars represents percentage change in that specific line due to K deficiency. Values of the top of the bars are showing per cent reduction due to K deficiency. 

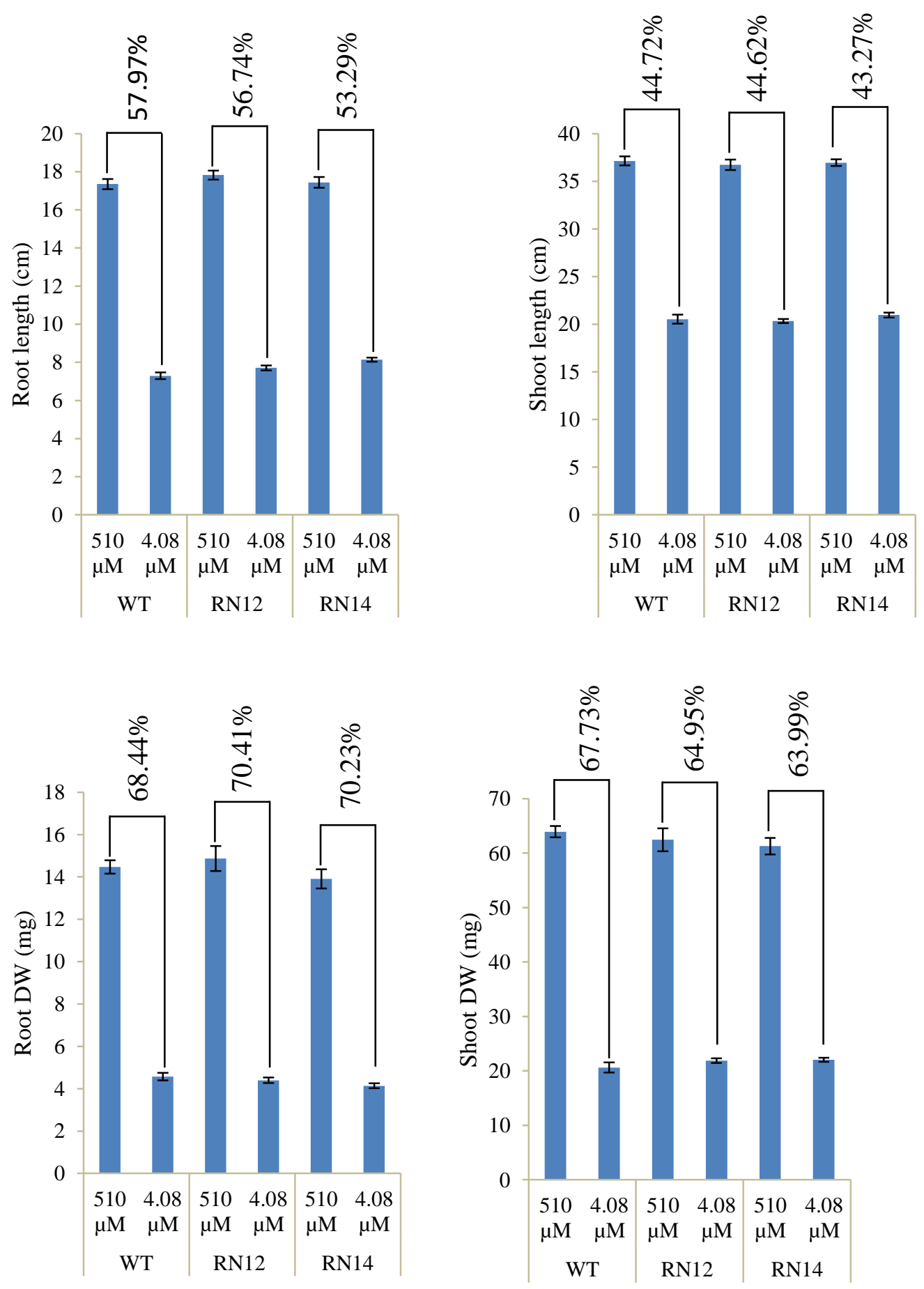

Figure S7. Effect of $O S J A Z 9$ knockdown on growth parameters of rice during K sufficient and deficient conditions. Growth parameters (length and dry biomass of root and shoot) of WT and OsJAZ9 RNAi (RN12 and RN14) lines under K deficient and sufficient conditions. Each bar represents average of 12 seedlings with standard error while the values above the bars represents percentage change in that specific line due to K deficiency. Values of the top of the bars are showing per cent reduction due to K deficiency. 
(A)
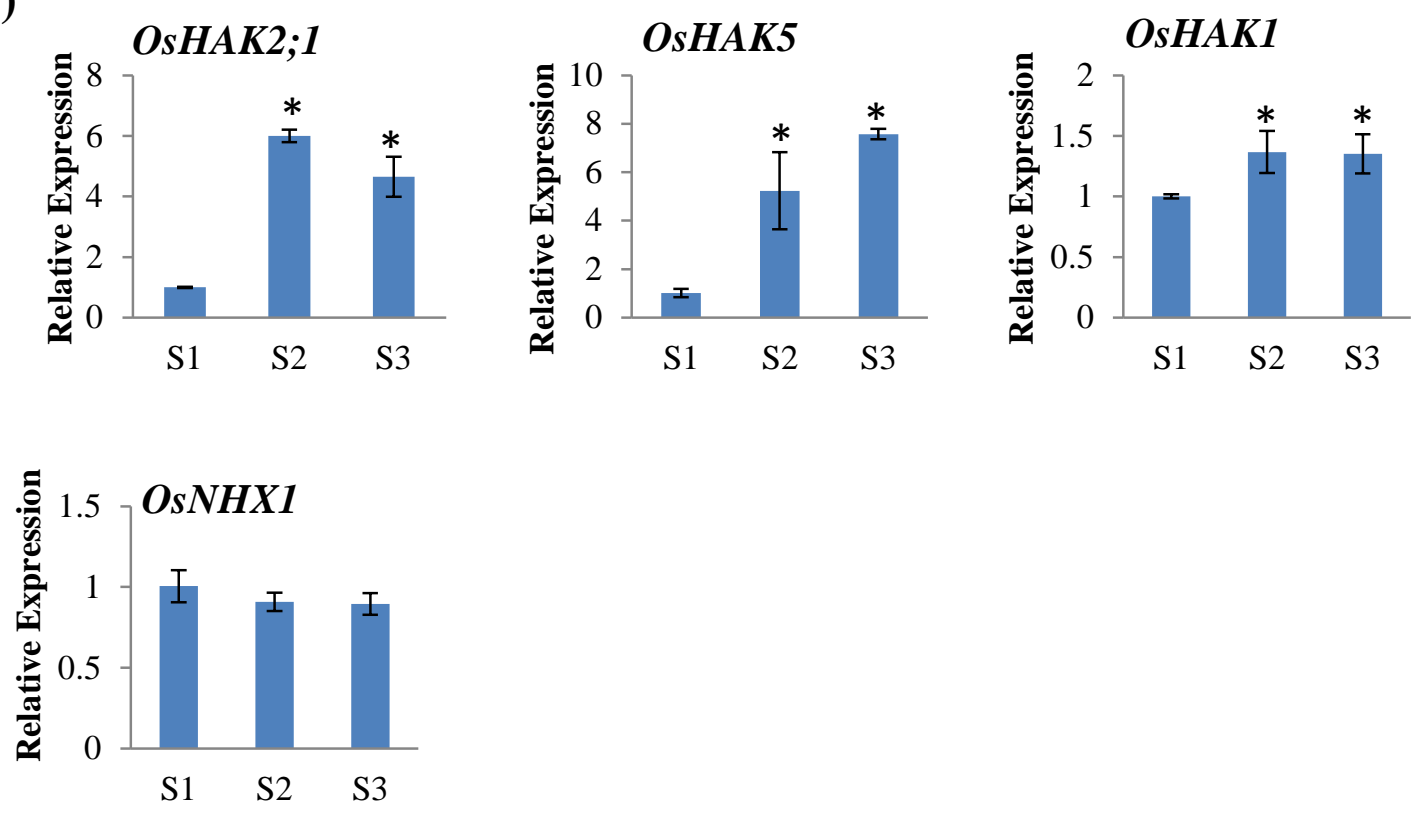

(B)
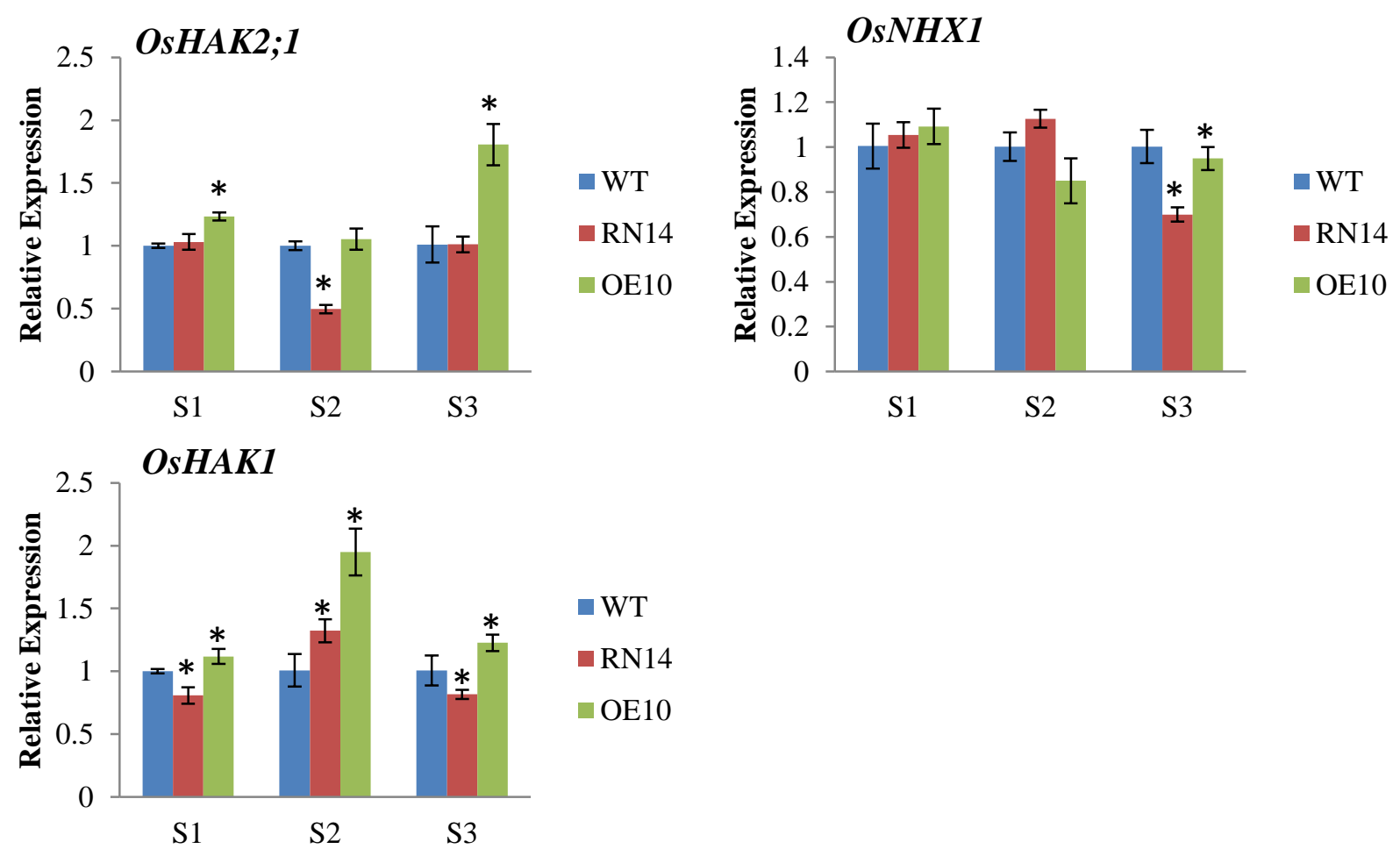

Figure S8. Overexpression of $O_{\boldsymbol{S} J A Z 9}$ enhances expression of $\mathrm{K}^{+}$transporters. (A) $\mathrm{K}^{+}$transporter encoding genes in WT under S2 (K deficiency, 15 days) and S3 (K recovery, $12 \mathrm{hrs)} \mathrm{treatments} \mathrm{with}$ respect to $\mathrm{S} 1$ (K sufficient). (B) Relative expression levels of OsHAK2;1, OsNHX1 and OsHAK1 in OsJAZ9 transgenics with respect to WT during S1, S2 and S3 treatments. Bars represent average of three biological replicates with standard error. Asterisk indicates significant change compared to WT of respective time point ( $p \leq 0.05$, Student's $t$-test). 

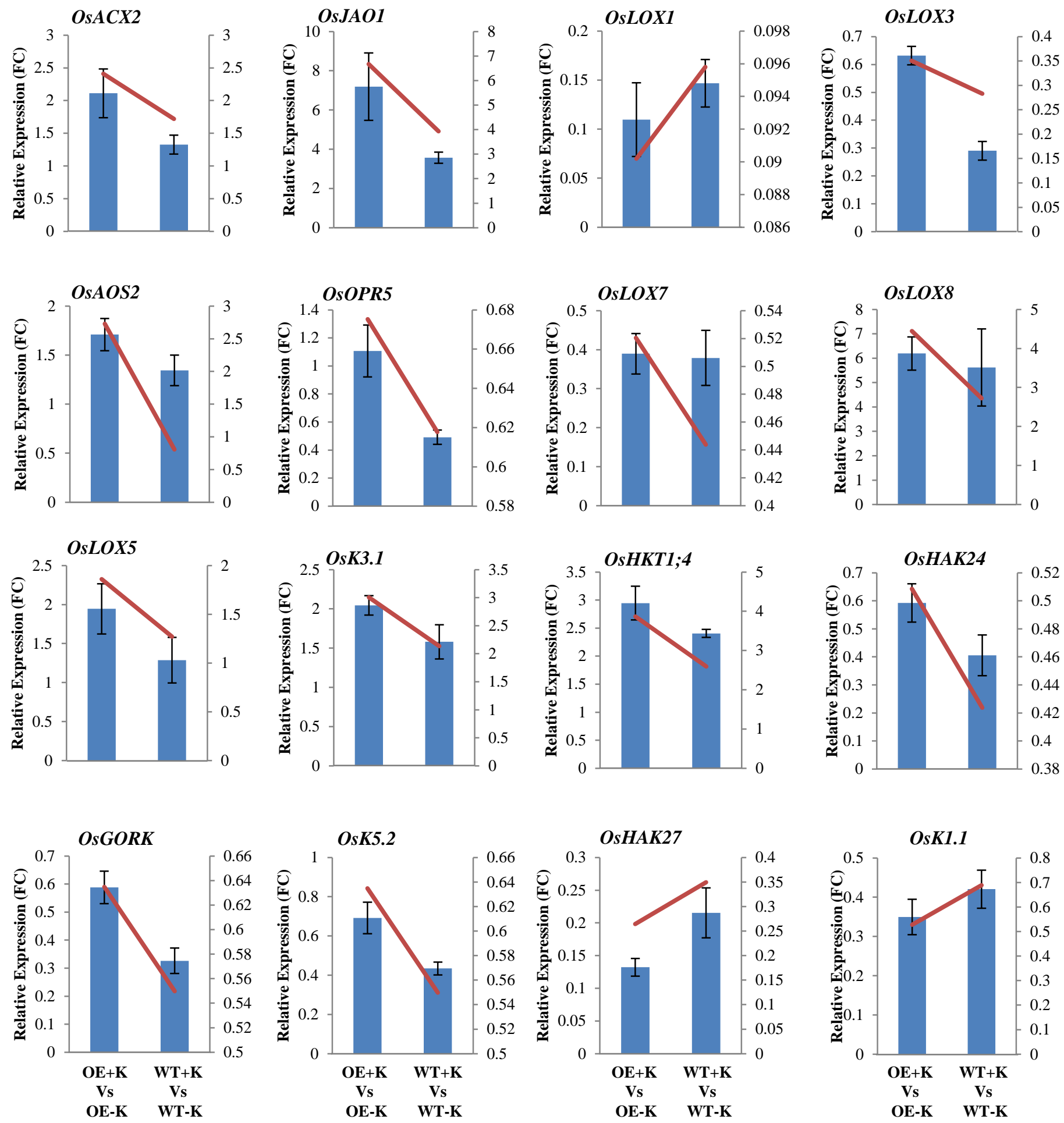

Figure S9. Validation of RNA-seq data with qRT-PCR. Relative expression levels (fold changes; FCs) of DEGs (shown in table S2 and S3) were analysed in WT and OsJAZ9 OE line during K deficient conditions w.r.t their own controls. All expression levels were normalized with respect to endogenous control, OsAct 1 and FCs were determined by $2-\Delta \Delta \mathrm{CT}$ method. Bar Graphs shows the expression obtained using qRT-PCR (right scale) and line graphs (left scale) shows the expression pattern obtained in RNAseq data. 

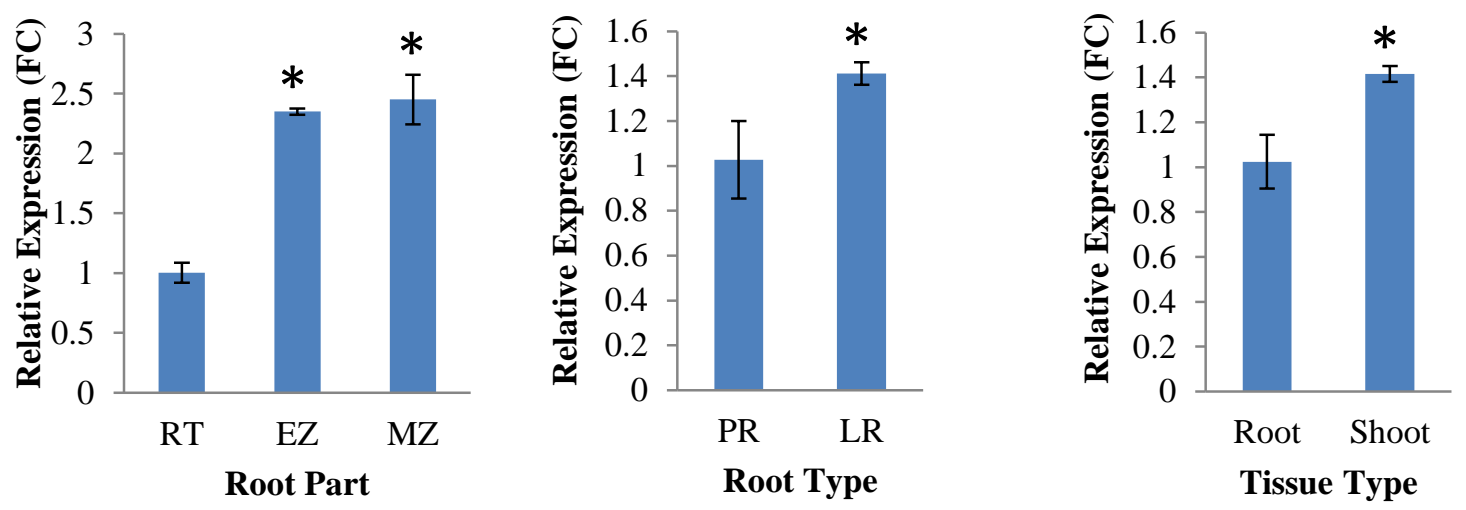

Figure S10. Tissue-specific expression of OsJAZ9. Expression of $O S J A Z 9$ shown by qRT-PCR analysis in different parts of root (Root Tip, RT; Elongation Zone, EZ; Maturation Zone, MZ), different root types (Primary Root, PR; Lateral Root, LR) and different tissues (Root and shoot). Each bar shows average fold change normalized to RT, PR and Root in respective graphs. $n \geq 3$, error bar represents standard error, asterisk marks the significant change with Student's $t$-test, $\mathrm{p} \leq 0.05$. 
(A)

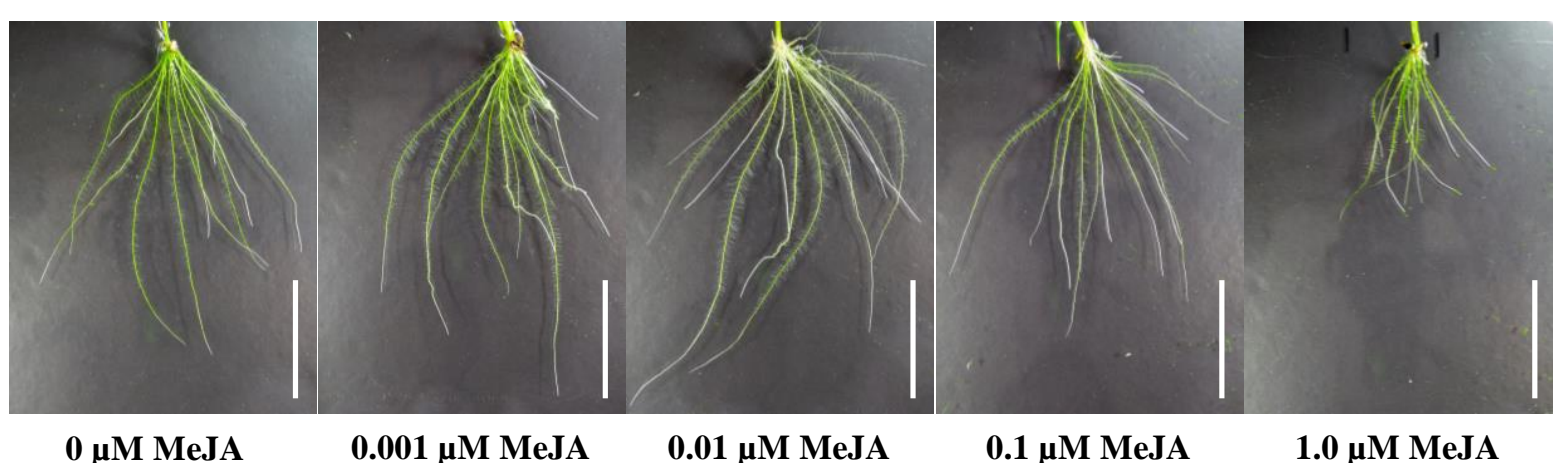

(B)

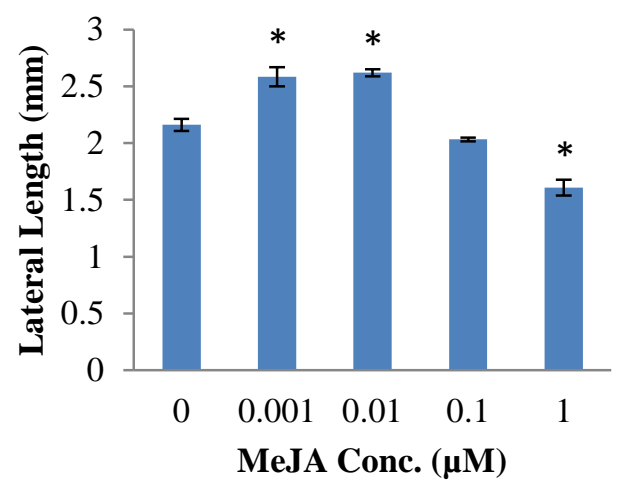

(C)

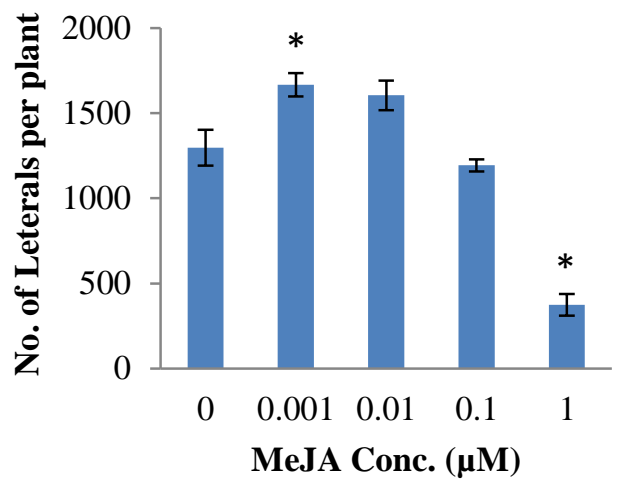

(D)

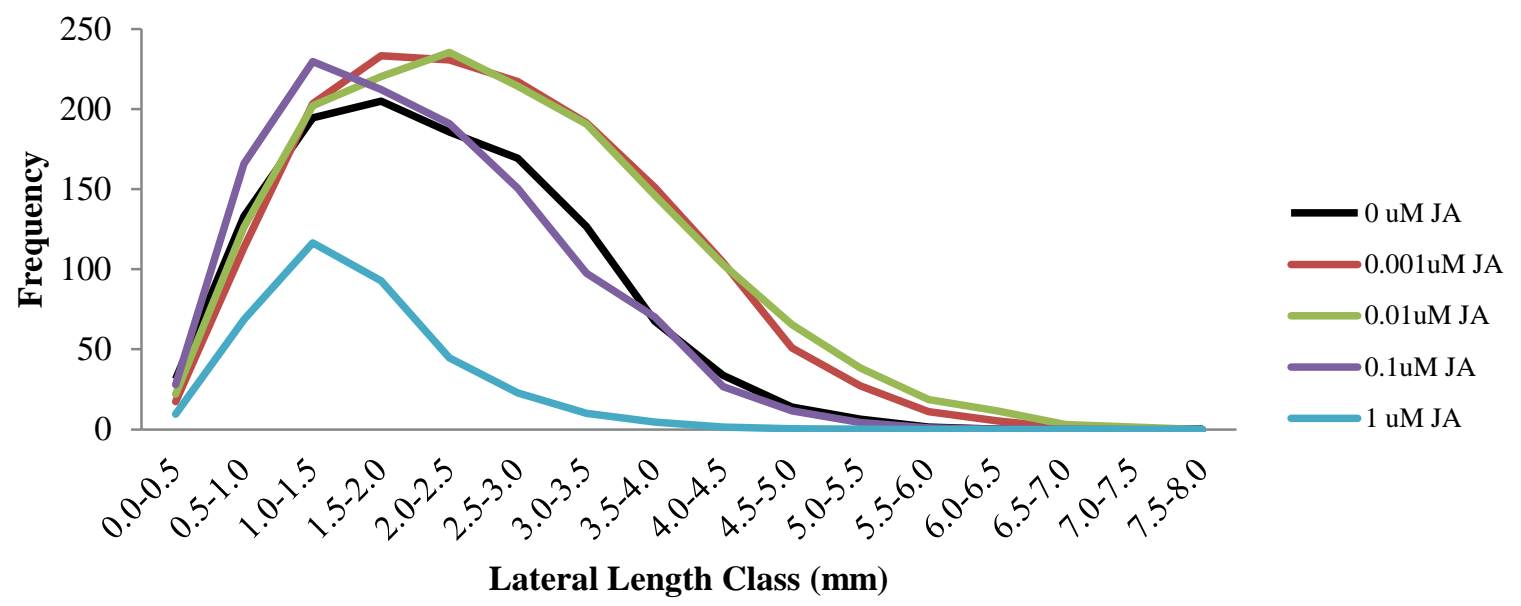

Figure S11. Exogenous application of MeJA modulates root system architecture under K sufficient conditions. (A) Representative image of 15-days-old WT plant roots grown with $510 \mu \mathrm{M}$ $\mathrm{K}_{2} \mathrm{SO}_{4}$ and different concentrations of exogenous MeJA (0, 0.001, 0.01, 0.1 and 1 $\left.\mu \mathrm{M}\right)$. (B) Average lateral length, (C) Average number of laterals per plant and (D) Frequency distribution curve showing abundance of different classes of laterals during the MeJA treatment for 15 days. Bar represents average of 4 seedlings (taking average of all the laterals in one plant as one replicate) with standard error. Frequency distribution graph shows the average distribution of the classes in 3 plants. Asterisks indicate significant changes in transgenics compared to WT at respective time points $(\mathrm{p} \leq 0.05$, Student's $t$-test). Scale $=5 \mathrm{~cm}$. 
(A)

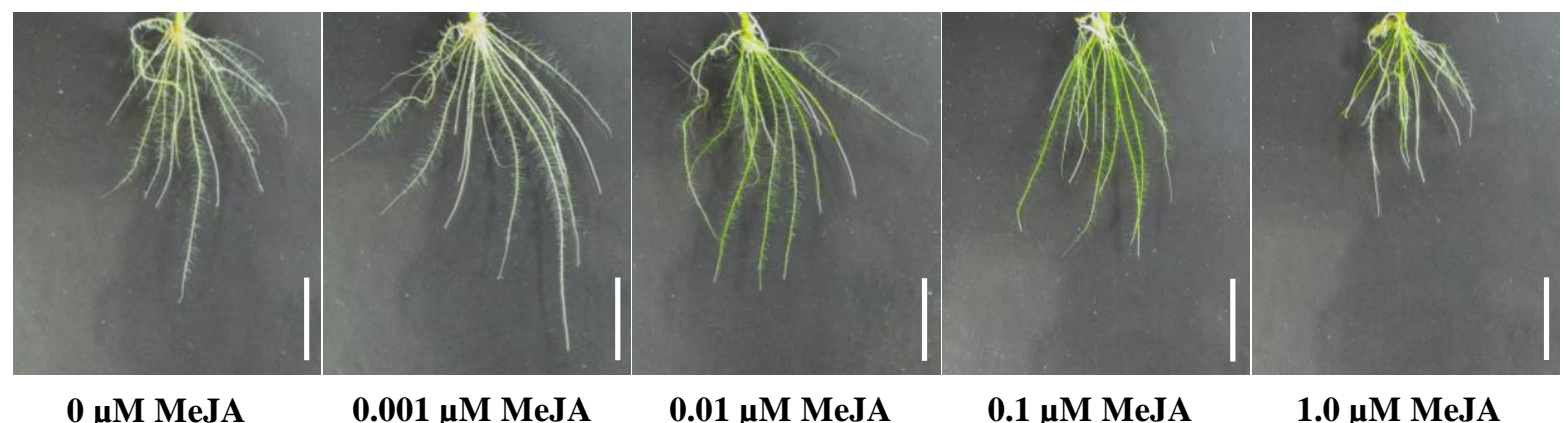

(B)

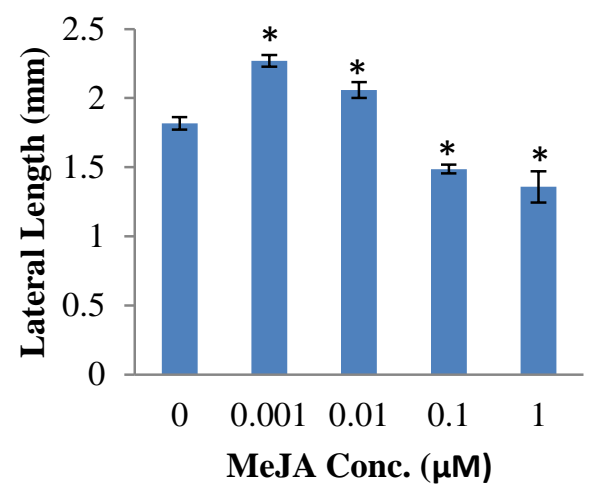

(C)

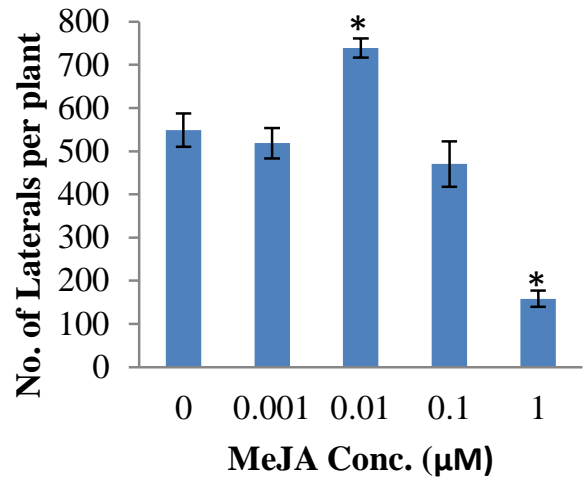

(D)

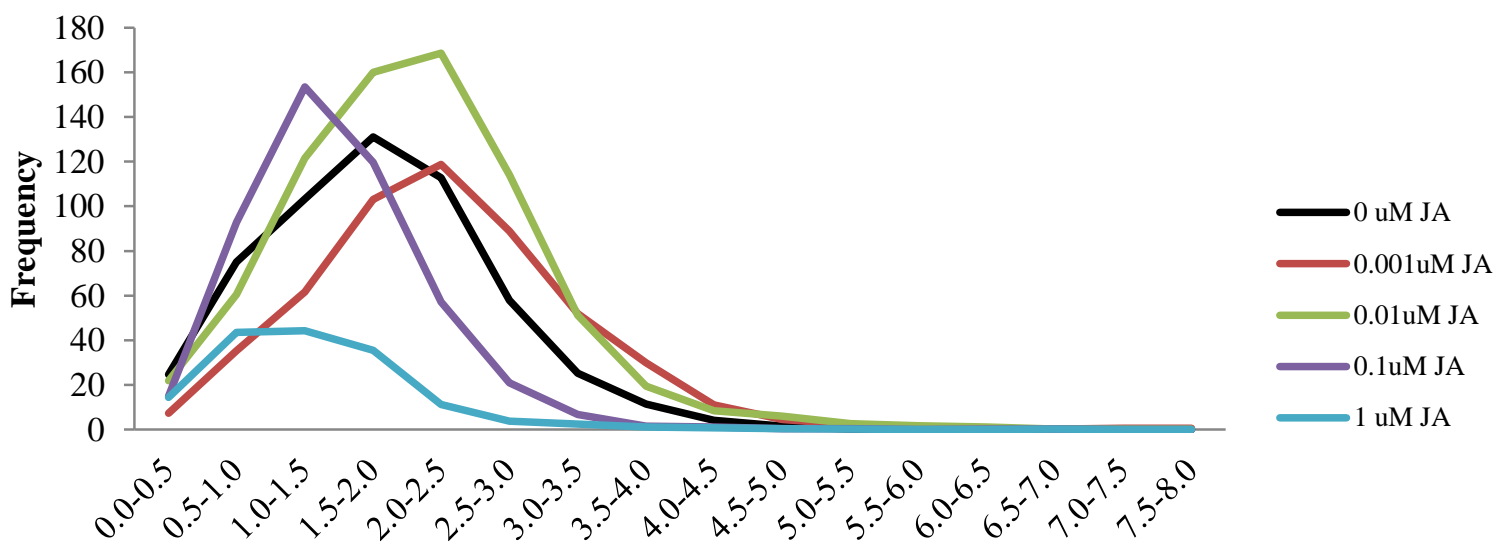

Lateral Length Class (mm)

Figure S12. Exogenous application of MeJA modulates root system architecture under $\mathrm{K}$ deficient conditions. (A) Representative image of 15-days-old WT plant roots grown with $4.08 \mu \mathrm{M}$ $\mathrm{K}_{2} \mathrm{SO}_{4}$ and different concentrations of exogenous MeJA (0, 0.001, 0.01, 0.1 and $\left.1 \mu \mathrm{M}\right)$. (B) Average lateral length, (C) Average number of laterals per plant and (D) Frequency distribution curve showing abundance of different classes of laterals during the MeJA treatment for 15 days. Bar represents average of 4 seedlings (taking average of all the laterals in one plant as one replicate) with standard error. Frequency distribution graph shows the average distribution of the classes in 4 plants. Asterisks indicate significant changes in transgenics compared to WT at respective time points $(\mathrm{p} \leq 0.05$, Student's $t$-test). Scale $=2 \mathrm{~cm}$. 
(A)

pZmUbi:OsJAZ9

(B)

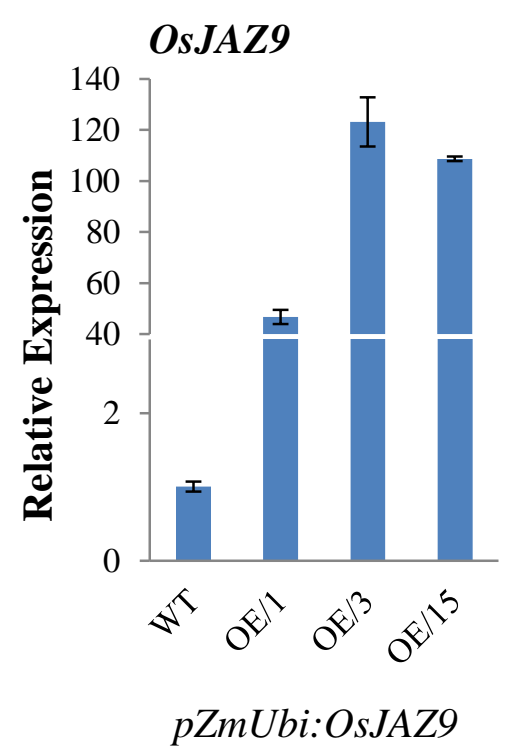

Figure S13. Raising and screening of OsJAZ9 OE rice transgenic lines with ZmUbil promoter. (A) Schematic of construct used for OsJAZ9 overexpression. (B) Relative expression of OsJAZ9 in overexpression lines. Gene expression analysis was done with qRT-PCR with at least three independent replicates. Asterisk indicates significant change compared to WT ( $p \leq 0.05$, Student's $t$-test). 

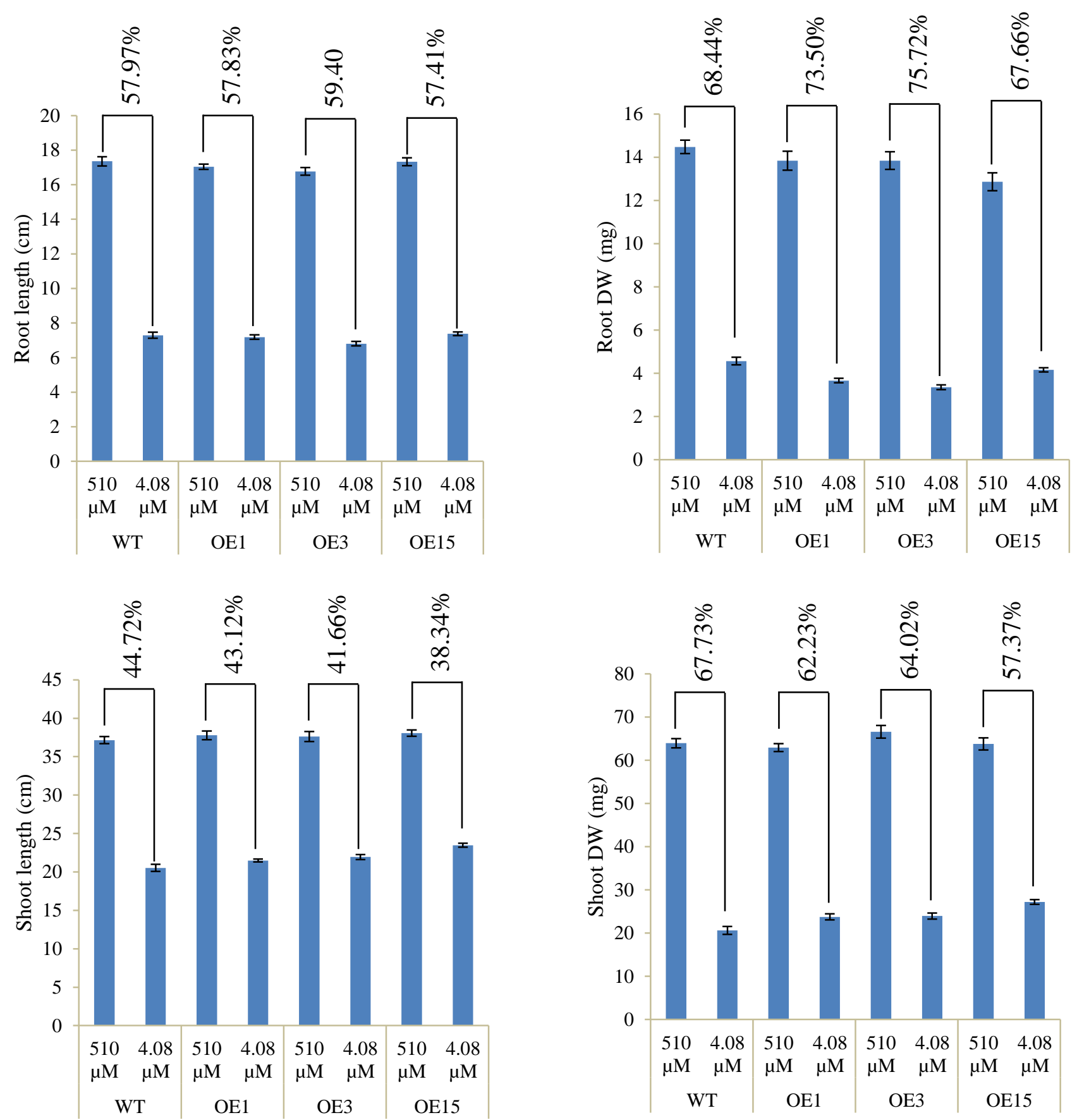

Figure S14. OsJAZ9 is involved in K deficiency tolerance in rice. Growth parameters (length and dry biomass of root and shoot) of WT and OsJAZ9 OE (OE1, OE3 and OE15) lines under K deficient and sufficient conditions. Each bar represents average of 12 seedlings with standard error while the values above the bars represents percentage change in that specific line due to $\mathrm{K}$ deficiency. 
(A)

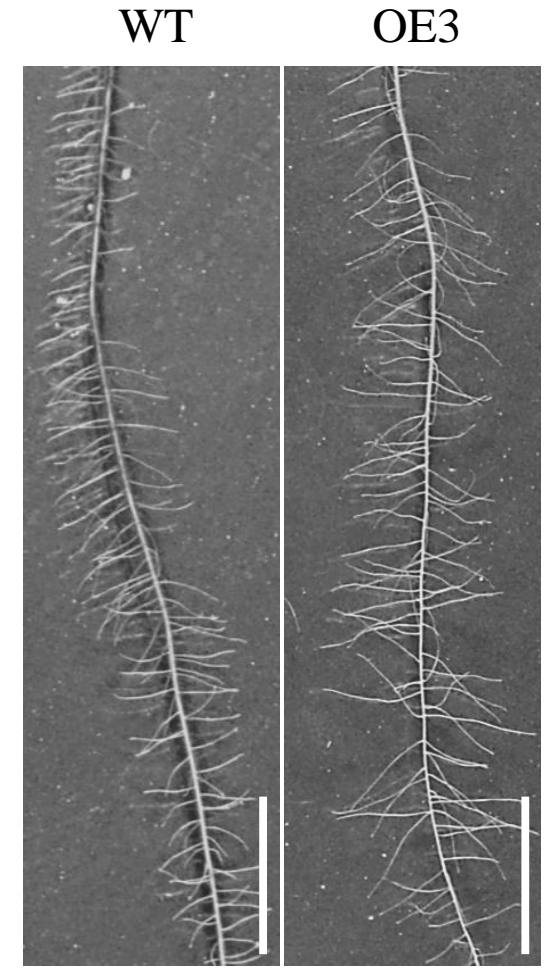

WT

OE3

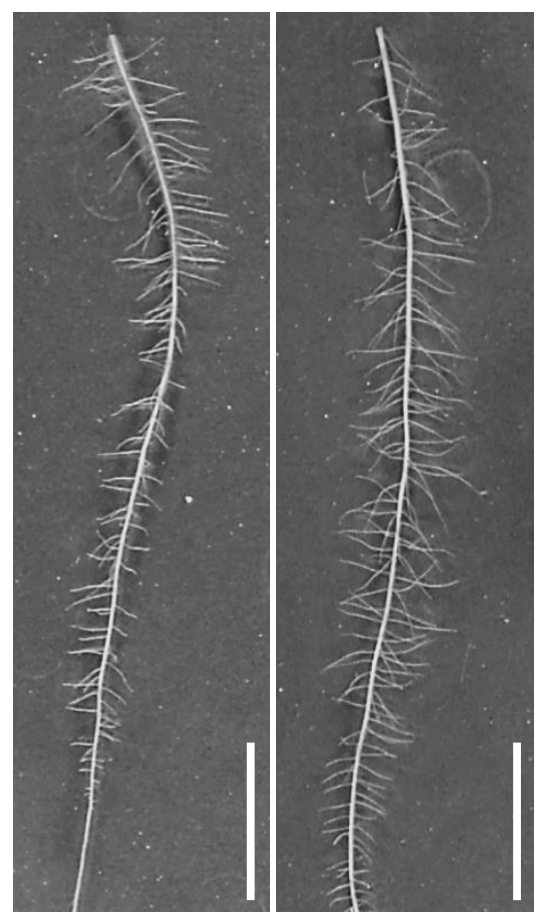

(B)
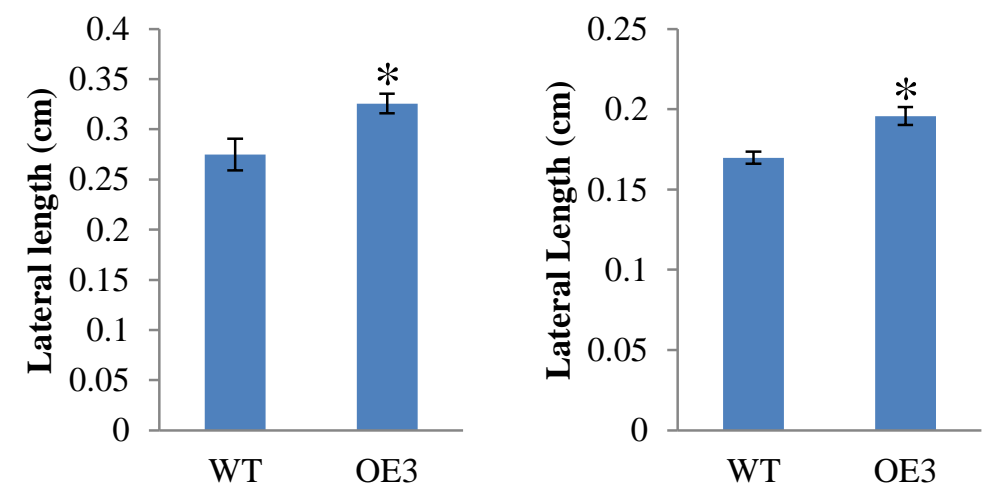

(C)
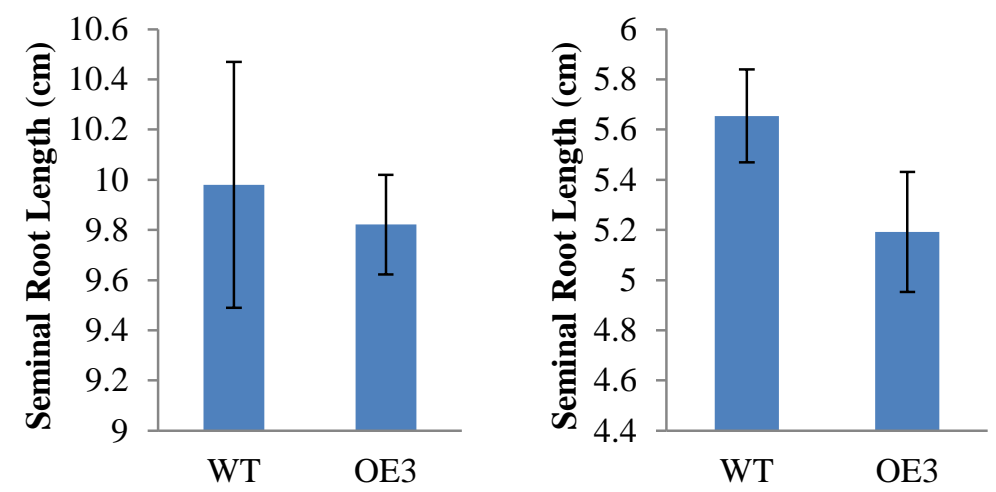

Figure S15. OsJAZ9 overexpression influences root system architecture. (A) Representative root images of 12-days-old WT and OSJAZ9 OE plants showing lateral root lengths during K sufficient (left panel) and $\mathrm{K}$ deficient conditions (right panel). (B) Average lateral root length and (C) Average seminal root length of WT and OsJAZ9 OE plants during $\mathrm{K}$ deficient and $\mathrm{K}$ sufficient conditions. Four independent plants were used for the analysis. Scale bar represents $1 \mathrm{~cm}$ while error bar represents SE among the replicates. ( $p \leq 0.05$, Student's $t$-test). 


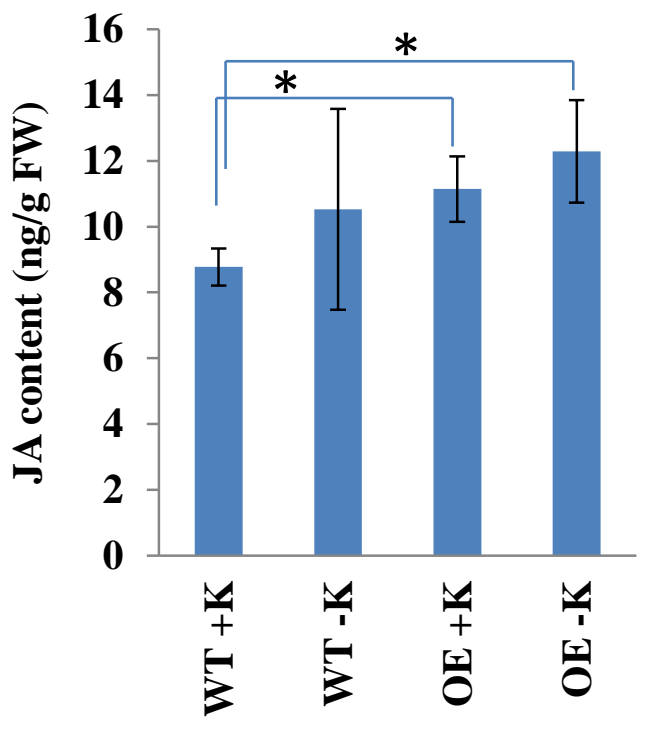

Figure S16. JA content in WT and OSJAZ9 OE (OE/10) plants during normal (+K) and 15 days of $\mathbf{K}$ deficiency (-K) conditions. Bars represent the average change in JA content among at least four replicates. Error bar represents SE of all the replicates, asterisk marks the significant change with Student's $t$-test, $\mathrm{p} \leq 0.05$. 


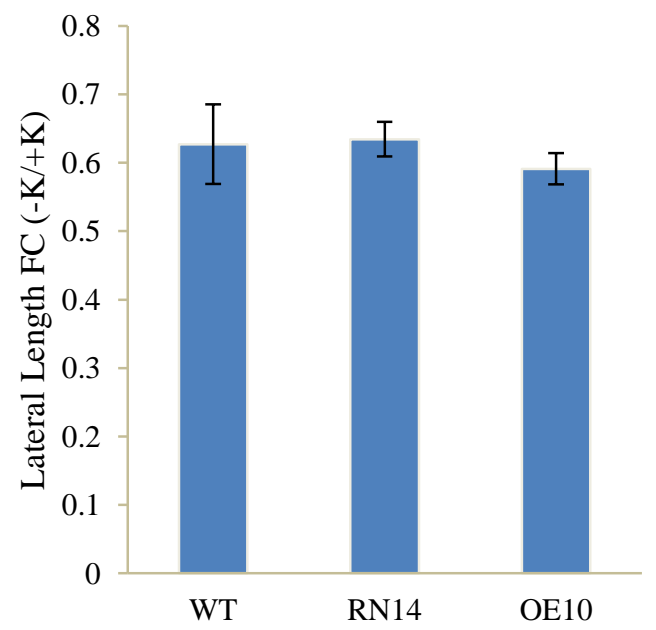

Figure X. OsJAZ9 overexpression influences root system architecture. Average fold change in lateral root length of plants (WT, OsJAZ9 RNAi and overexpression) grown under $-\mathrm{K} /+\mathrm{K}$ (K deficient/K sufficient) conditions. Four independent plants were used for the analysis. Error bar represents SE among the replicates. 
Table S1 List of primers used for qRT-PCR and gene cloning

\begin{tabular}{|c|c|c|}
\hline \multicolumn{3}{|c|}{ Primer used for qRT-PCR } \\
\hline Primer ID & Forward Primer & Reverse Primer \\
\hline qOsUBQ5 & ACCACTTCGACCGCCACTACT & ACGCCTAAGCCTGCTGGTT \\
\hline OsActin1 & CAGCCACACTGTCCCCATCTA & AGCAAGGTCGAGACGAAGGA \\
\hline qOsACX2 & CATGTGGTGGCCATGGGTAT & TCATGATCATTGCGCAAAGC \\
\hline qOsGORK & CGTGGCCTACCGTGATCCT & GCGATGGAGGTGGGATTG \\
\hline qOsHAK24 & CAAGGGAGGCAGGCATGT & CGCTTTTCGCCTTCATATGG \\
\hline qOsHAK27 & ACCGGCCATTTCAGTTCTGT & CAGGTTCGGTGCTTTCTCTTTC \\
\hline qOsHKT1;4 & TCCAGACCATCTCTGCACAGAT & GTTTGCCAGAGTCGCTCCAT \\
\hline qOsJAO1 & CCCGACGCCTTCATCGT & GTACATCGAGTTGCTCAAGATCTCA \\
\hline qOsK5.2 & CGTGGCCTACCGTGATCCT & GCGATGGAGGTGGGATTG \\
\hline qOsLOX1 & CATGCCGTCCAAGATGCA & GGAGTGCGACGACAGGATGT \\
\hline qOsLOX3 & GCTGGTCTGAATCCCATGGT & TCAAGAGTACTTTTTGGTGGGAATT \\
\hline qOsLOX5 & GGGCCTCACGGTGCAA & TCGTGGTGGTCTAGGATGAAAA \\
\hline qOsLOX7 & CCGATATCTATGCCCATTGGA & CCATCCCCCTCTTGATGAGA \\
\hline qOsLOX8 & GCAGCTCAGCGAGATGCA & CGTTGATCCGCATCGTGTAC \\
\hline qOsOPR5 & TGATGCGCCATTGAACAAGT & TCCAACAATTGGGTCTTGAGTGT \\
\hline qOsK3.1 & GGCATCCTCCGGCTATGG & TGATGTCCTTCTCCAGCCTTGT \\
\hline qOsK1.1 & TTGCGTTTGAATCGTACAGC & CCTTTACGACACCAGCCATT \\
\hline qOsJAZ9 & TCGACGGATCCCATGACC & GGCCTTGACGTACTGGCTG \\
\hline OsJAZ9_RNAi & CGTACAGTACAGATCGTTCGCAAT & GGACAACTAAGCACAAGCACAAA \\
\hline $\mathrm{qOsCOI} 1 \mathrm{~b}$ & AGGATGCCGTGAACTGGAAT & TCCAGGGCCCCATTTGT \\
\hline qOsMYC2 & CTAGCGAGGAAACCCAATCG & CCATCCATCCATCCTAАСАC \\
\hline qOSAOS1 & CGAGCTCTTCCTCCGATACG & GTCAGAAGGTGGCCTTCTTGAG \\
\hline qOsAOS2 & GGAGGAAGCTGCTGCAATAC & GGAGGTTGAAGCTTTGGTGA \\
\hline OsJAR1 & GGTTTGTGAACCCATCAAACAGC & CAAAATCCATAGGCAGTACTG \\
\hline qOsHAK1 & GTTGATGATGCTGATGTTGGAAG & CCAACACTTTCAGCTGAAAC \\
\hline qOsHKT2;1 & CACAGTCTCCTCGTTTGCGAA & GCAAGAATCTGGCCGATGAA \\
\hline qOsHAK5 & CATTGTGGACTATTTTGAAAGAA & GGAGAACTACAGAAAAGCCAATC \\
\hline qOsNHX1 & TCCAGCCTCCGGATGCT & ATCAGCGCGTCGTCGAA \\
\hline \multicolumn{3}{|c|}{ Primers used for cloning } \\
\hline OsJAZ9_OE & CACCATCAGCGACGTACAGTACA & CGCCGCGATAACTAGGGTAAC \\
\hline OsJAZ9_RNAi & CACCGACACATGCACTC & CAGAACAGTCGAAGGAATTTTA \\
\hline OsJAZ9_GUS & TGCTTAAGATCTAATGGCGTCGACGGAT & TGCTTAAGATCTACGCGCGAGTGCATGTG \\
\hline OsJAZ9AC_GUS & TGCTTAAGATCTAATGGCGTCGACGGAT & TGCTTAAGATCTACCATGTCCACCAGCGC \\
\hline pOsJAZ9_Gus & CACCAGTGCGCACCATAGTTC & TGCCGTCTTCTAGCACGGAC \\
\hline
\end{tabular}


Table S2. Effect of K deficiency on expression of JA associated genes.

\begin{tabular}{lllccll}
\hline $\begin{array}{l}\text { Gene } \\
\text { Category }\end{array}$ & MSU ID & $\begin{array}{l}\text { Gene } \\
\text { Name }\end{array}$ & $\begin{array}{c}\text { WT+K Vs } \\
\text { WT-K }\end{array}$ & $\begin{array}{c}\text { P } \\
\text { Value }\end{array}$ & Q Value & GOSlime Annotation \\
\hline & Os02g12680 & AOS3 & 2.5484 & 0.0017 & 0.0130 & cytochrome P450, putative, expressed \\
& Os08g39850 & OsLOX8 & 2.7217 & 0.0001 & 0.0007 & lipoxygenase, chloroplast precursor, putative, expressed \\
& Os11g39220 & OsACX2 & 1.7181 & 0.0001 & 0.0012 & acyl-coenzyme A oxidase, putative, expressed \\
& Os01g27230 & OPR10 & 1.8214 & 0.0002 & 0.0022 & $\begin{array}{l}\text { 12-oxophytodienoate reductase, putative, expressed } \\
\text { flavonol synthase/flavanone 3-hydroxylase, putative, } \\
\text { expressed }\end{array}$ \\
& Os01g61610 & JAO1 & 3.9358 & 0.0001 & 0.0007 & HAD superfamily phosphatase, putative, expressed \\
\hline
\end{tabular}


Table S3. Effect of OsJAZ9 expression on expression of JA associated genes under K deficiency.

\begin{tabular}{|c|c|c|c|c|c|c|c|c|c|}
\hline \multirow[b]{2}{*}{$\begin{array}{l}\text { Gene } \\
\text { Category }\end{array}$} & \multirow[b]{2}{*}{ MSU ID } & \multirow[b]{2}{*}{$\begin{array}{l}\text { Gene } \\
\text { Name }\end{array}$} & \multicolumn{2}{|c|}{ Fold Change } & \multicolumn{2}{|c|}{ OE+K Vs OE -K } & \multicolumn{2}{|c|}{ WT+K Vs WT-K } & \multirow[b]{2}{*}{ GOSlime Annotation } \\
\hline & & & $\begin{array}{c}\text { OE+K } \\
\text { Vs } \\
\text { OE -K }\end{array}$ & $\begin{array}{c}\text { WT+K } \\
\text { Vs } \\
\text { WT-K } \\
\end{array}$ & $\begin{array}{c}\mathbf{P} \\
\text { value }\end{array}$ & $\begin{array}{c}\mathbf{Q} \\
\text { Value } \\
\end{array}$ & $\begin{array}{c}\mathbf{P} \\
\text { Value } \\
\end{array}$ & $\begin{array}{c}\mathbf{Q} \\
\text { Value } \\
\end{array}$ & \\
\hline & Os03g12500 & AOS2 & 2.7263 & NS & 0.0001 & 0.0007 & 0.3161 & 0.6305 & cytochrome P450, putative, expressed \\
\hline & Os02g12680 & AOS3 & 5.4734 & 2.5484 & 0.0001 & 0.0007 & 0.0017 & 0.0130 & cytochrome $\mathrm{P} 450$, putative, expressed \\
\hline & Os02g12690 & AOS4 & 0.1591 & 0.3320 & 0.0001 & 0.0007 & 0.0001 & 0.0012 & cytochrome P450, putative, expressed \\
\hline & Os03g32314 & $A O C$ & 0.6586 & 0.6601 & 0.0026 & 0.0186 & 0.0026 & 0.0189 & $\begin{array}{l}\text { allene oxide cyclase } 4 \text {, chloroplast precursor, putative, } \\
\text { expressed }\end{array}$ \\
\hline & Os02g43700 & $D A D 1$ & 0.4736 & 0.6386 & 0.0002 & 0.0017 & 0.0059 & 0.0367 & lypoxygenase \\
\hline & Os02g10120 & OsLOX1 & 0.0902 & 0.0958 & 0.0001 & 0.0007 & 0.0001 & 0.0007 & lipoxygenase, putative, expressed \\
\hline & Os12g37260 & $L O X 2$ & 0.4736 & NS & 0.0061 & 0.0376 & 0.1148 & 0.3374 & $\begin{array}{l}\text { lipoxygenase } 2.1 \text {, chloroplast precursor, putative, } \\
\text { expressed }\end{array}$ \\
\hline & Os03g49260 & $L O X 3$ & 0.3508 & 0.2829 & 0.0001 & 0.0007 & 0.0001 & 0.0007 & lipoxygenase, putative, expressed \\
\hline & Os03g49380 & OsLOX5 & 1.8601 & NS & 0.0001 & 0.0007 & 0.1102 & 0.3279 & lipoxygenase, putative, expressed \\
\hline & Os05g23880 & OsLOX7 & 0.5205 & 0.4439 & 0.0001 & 0.0007 & 0.0001 & 0.0007 & lipoxygenase, putative, expressed \\
\hline & Os08g39850 & $\begin{array}{l}\text { OsLOX8 } \\
\text { OsLOXL- }\end{array}$ & 4.4474 & 2.7217 & 0.0001 & 0.0007 & 0.0001 & 0.0007 & lipoxygenase, chloroplast precursor, putative, expressed \\
\hline & Os03g52860 & 2 & 0.2465 & 0.3115 & 0.0001 & 0.0007 & 0.0001 & 0.0007 & lipoxygenase, putative, expressed \\
\hline & Os11g39220 & $O s A C X 2$ & 2.4093 & 1.7181 & 0.0001 & 0.0007 & 0.0001 & 0.0012 & acyl-coenzyme A oxidase, putative, expressed \\
\hline & Os06g20920 & $J M T$ & 0.5246 & 0.4895 & 0.0058 & 0.0361 & 0.0015 & 0.0121 & expressed \\
\hline & Os06g11210 & OPR5 & NS & 0.6178 & 0.0183 & 0.0885 & 0.0034 & 0.0236 & 12-oxophytodienoate reductase, putative, expressed \\
\hline JA & Os01g27230 & OPR10 & 1.6846 & 1.8214 & 0.0005 & 0.0049 & 0.0002 & 0.0022 & 12-oxophytodienoate reductase, putative, expressed \\
\hline $\begin{array}{l}\text { Biosynthe } \\
\text { sis genes }\end{array}$ & Os01g61610 & $J A O 1$ & 6.6732 & 3.9358 & 0.0002 & 0.0022 & 0.0001 & 0.0007 & $\begin{array}{l}\text { flavonol synthase/flavanone 3-hydroxylase, putative, } \\
\text { expressed }\end{array}$ \\
\hline $\begin{array}{c}\text { JA } \\
\text { Signaling }\end{array}$ & & & & & & & & & \\
\hline Genes & Os $10 \mathrm{~g} 25290$ & $J A Z 12$ & 2.7992 & NS & 0.0001 & 0.0007 & 0.0113 & 0.0612 & ZIM domain containing protein, putative, expressed \\
\hline $\begin{array}{c}\text { JA } \\
\text { Marker }\end{array}$ & Os05g46480 & LEA3 & 22.0807 & NS & 0.0001 & 0.0007 & 0.0001 & 0.0007 & $\begin{array}{l}\text { late embryogenesis abundant protein, group } 3 \text {, putative, } \\
\text { expressed }\end{array}$ \\
\hline Genes & Os05g10210 & $V S P 2$ & 4.1088 & 1.6605 & 0.0001 & 0.0007 & 0.0012 & 0.0101 & HAD superfamily phosphatase, putative, expressed \\
\hline
\end{tabular}


Table S4. Effect of OSJAZ9 expression on K transporter expression under K deficiency

\begin{tabular}{|c|c|c|c|c|c|c|c|c|c|}
\hline \multirow{7}{*}{$\begin{array}{l}\text { Family } \\
\text { KUP/HAK/KT } \\
\text { family }\end{array}$} & \multirow[b]{2}{*}{ MSU ID } & \multirow[b]{2}{*}{ Gene Name } & \multicolumn{2}{|c|}{ Fold Change } & \multicolumn{2}{|c|}{ OE+K Vs OE -K } & \multicolumn{2}{|c|}{ WT+K Vs WT-K } & \multirow[b]{2}{*}{ UP/DN } \\
\hline & & & $\begin{array}{c}\text { OE+K Vs } \\
\text { OE -K }\end{array}$ & $\begin{array}{c}\text { WT+K Vs } \\
\text { WT-K }\end{array}$ & $\begin{array}{c}\mathbf{P} \\
\text { VALUE }\end{array}$ & $\begin{array}{c}\mathbf{Q} \\
\text { VALUE }\end{array}$ & $\begin{array}{c}\mathbf{P} \\
\text { VALUE }\end{array}$ & $\begin{array}{c}\mathbf{Q} \\
\text { VALUE }\end{array}$ & \\
\hline & Os01g70490 & OsHAK5 & 2.05340 & 3.00194 & 0.0001 & 0.0007 & 0.0001 & 0.0007 & $\mathrm{DN}$ \\
\hline & Os06g42030 & OsHAK10 & 0.43768 & 0.37120 & 0.0001 & 0.0007 & 0.0001 & 0.0007 & UP \\
\hline & Os09g27580 & OsHAK17 & 0.51064 & 0.60262 & 0.0001 & 0.0007 & 0.0007 & 0.0061 & $\mathrm{DN}$ \\
\hline & Os06g15910 & OsHAK 24 & 0.50844 & 0.42377 & 0.0002 & 0.0017 & 0.0001 & 0.0007 & UP \\
\hline & Os03g37830 & OsHAK27 & 0.26458 & 0.34944 & 0.0001 & 0.0007 & 0.0001 & 0.0007 & $\mathrm{DN}$ \\
\hline \multirow[t]{3}{*}{ AKT family } & Os01g45990 & OsK1.1 & 0.52729 & 0.68904 & 0.0001 & 0.0007 & 0.0080 & 0.0465 & $\mathrm{DN}$ \\
\hline & Os05g35410 & OsK3.1 & 3.00689 & 2.13439 & 0.0001 & 0.0007 & 0.0001 & 0.0012 & UP \\
\hline & Os06g14030 & OsK5.2 & 0.63491 & 0.54976 & 0.0051 & 0.0327 & 0.0002 & 0.0017 & UP \\
\hline \multirow[t]{3}{*}{ Trk family } & Os06g48810 & OsHKT2;2 & 4.73023 & 5.67469 & 0.0001 & 0.0007 & 0.0001 & 0.0007 & $\mathrm{DN}$ \\
\hline & Os04g51830 & OsHKT1;4 & 3.86436 & 2.59602 & 0.0012 & 0.0101 & 0.0087 & 0.0497 & UP \\
\hline & Os01g20160 & OsHKT1;5 & 0.19389 & 0.25082 & 0.0037 & 0.0253 & 0.0067 & 0.0406 & $\mathrm{DN}$ \\
\hline \multirow[t]{2}{*}{$\begin{array}{l}\text { Other K } \\
\text { transporters }\end{array}$} & Os12g44360 & SOS1 & 0.52463 & 0.47277 & 0.0001 & 0.0007 & 0.0001 & 0.0007 & UP \\
\hline & Os06g14030 & GORK & 0.63491 & 0.54976 & 0.0051 & 0.0327 & 0.0002 & 0.0017 & UP \\
\hline
\end{tabular}


Table S5. Effect of $O s J A Z 9$ expression on expression of $O s J A Z$ genes.

\begin{tabular}{|c|c|c|c|c|c|c|c|c|c|}
\hline \multirow[b]{2}{*}{ Category } & \multirow[b]{2}{*}{ MSU ID } & \multirow[b]{2}{*}{$\begin{array}{c}\text { Gene } \\
\text { Name }\end{array}$} & \multicolumn{2}{|c|}{ Fold Change } & \multicolumn{2}{|c|}{ WT+K Vs OE+K } & \multicolumn{2}{|c|}{ WT-K Vs OE-K } & \multirow[b]{2}{*}{ GOSlim Annotation } \\
\hline & & & $\begin{array}{l}\text { WT+K } \\
\text { Vs } \\
\text { OE+K }\end{array}$ & $\begin{array}{l}\text { WT-K } \\
\text { Vs } \\
\text { OE-K }\end{array}$ & P value & Q value & P value & $Q$ value & \\
\hline & Os04g55920 & $J A Z 1$ & 0.8657 & 0.7747 & 0 & 0.59 & 0.06 & 0 & zinc-finger protein, putative, expressed \\
\hline & Os07g05830 & $J A Z 2$ & 0.0027 & 0.3799 & 1 & 1 & 1 & 1 & zinc-finger protein, putative, expressed \\
\hline & Os08g33160 & $J A Z 3$ & 0.9049 & 0.9561 & 0 & 0.78 & 0.75 & 1 & ZIM motif family protein, expressed \\
\hline & Os09g23660 & $J A Z 4$ & 0.8980 & 0.8640 & 1 & 0.83 & 0.4 & 1 & ZIM motif family protein, expressed \\
\hline & Os04g32480 & $J A Z 5$ & 0.6802 & 0.7559 & 0 & 0.6 & 0.35 & 1 & \multirow{2}{*}{$\begin{array}{l}\text { zinc-finger protein, putative, expressed } \\
\text { ZIM domain containing protein, } \\
\text { putative, expressed }\end{array}$} \\
\hline & Os03g28940 & $J A Z 6$ & 0.5110 & 0.6006 & 0 & 0 & 0 & 0 & \\
\hline & Os07g42370 & $J A Z 7$ & 0.6822 & 0.6394 & 0 & 0.03 & 0 & 0 & \multirow{3}{*}{$\begin{array}{l}\text { zinc-finger protein, putative, expressed } \\
\text { zinc-finger protein, putative, expressed }\end{array}$} \\
\hline & Os09g26780 & $J A Z 8$ & 0.3315 & 0.3787 & 0 & 0 & 0 & 0 & \\
\hline & Os03g08310 & $J A Z 9$ & 204.2619 & 163.9600 & 0 & 0 & 0 & 0 & \\
\hline & Os03g08320 & $J A Z 10$ & 0.4277 & 0.6511 & 0 & 0 & 0.03 & 0 & \multirow{3}{*}{$\begin{array}{l}\text { ZIM domain containing protein, } \\
\text { putative, expressed } \\
\text { ZIM domain containing protein, } \\
\text { putative, expressed } \\
\text { ZIM domain containing protein, } \\
\text { putative, expressed }\end{array}$} \\
\hline & Os $10 \mathrm{~g} 25290$ & $J A Z 12$ & 0.3498 & 0.6259 & 0 & 0 & 0.01 & 0 & \\
\hline Repressor & Os $10 \mathrm{~g} 25230$ & $J A Z 13$ & 0.4824 & 0.3100 & 0 & 0.3 & 0.02 & 0 & \\
\hline
\end{tabular}

